\title{
A taxonomic synopsis of Acianthera (Orchidaceae: Pleurothallidinae) in Peru, including two new species
}

\author{
Alexander Damián ${ }^{1,2, *}$, Nicole Mitidieri ${ }^{3} \&$ Guy Chiron ${ }^{4}$ \\ ${ }^{1}$ Facultad de Ciencias Ambientales, Universidad Científica del Sur, Lima, Peru. \\ ${ }^{2,3}$ Posgrado en Botánica Tropical, Facultad de Ciencias Biológicas, Universidad Nacional Mayor de San Marcos, \\ Av. Venezuela, Cdra. 34 s.n., Lima, Peru. \\ ${ }^{4}$ Herbiers, Université de Lyon 1, F-69622 Villeurbanne Cedex, France. \\ *Author for correspondence: adamian.pz2@gmail.com, https://orcid.org/0000-0002-0233-9935 \\ 3nicole.mitidieri@unmsm.edu.pe, https://orcid.org/0000-0001-6045-4491 \\ ${ }^{4}$ g.r.chiron@wanadoo.fr, https://orcid.org/0000-0003-0971-4715
}

\begin{abstract}
A taxonomic synopsis of Peruvian Acianthera including 35 accepted species is presented. An artificial key and maps for known localities from all these species are also included. Acianthera berlinensis sp. nov. and A. marleniae sp. nov. are described and illustrated as new species based on specimens collected in the Peruvian regions of Amazonas and Cajamarca, respectively. Information on the distribution and habitat is provided for both new taxa, followed by a discussion comparing the traits that distinguish them from their closest relatives. A. consatae, A. dodsonii, A. lamia, A. scalpricaulis and A. toachica, previously known only from Ecuador and Bolivia, are herein reported for the first time for the Peruvian flora.
\end{abstract}

Keywords. Acianthera berlinensis sp. nov., Acianthera marleniae sp. nov., Amazonas, checklist, taxonomy.
Resumen. Se presenta una sinopsis taxonómica de Acianthera en Perú que incluye 35 especies. Se incluye una clave artificial y mapas para las localidades conocidas de todas las especies citadas. Acianthera berlinensis sp. nov. y A. marleniae sp. nov. se describen como nuevas especies, basadas en especímenes recolectados en los departamentos de Amazonas y Cajamarca. Se ofrece informacion de su distribución, hábitat y fenología para ambos taxones, seguido de una discusión sobre las características que las distinguen de especies similares. Se citan por primera vez para la flora peruana $A$. consatae, A. dodsonii, A. lamia, A. scalpricaulis y A. toachica, anteriormente conocidas solo en Ecuador y Bolivia.

Palabras clave. Acianthera berlinensis sp. nov., Acianthera marleniae sp. nov., Amazonas, lista taxonómica, taxonomía.

Damián A., Mitidieri N. \& Chiron G. 2018. A taxonomic synopsis of Acianthera (Orchidaceae: Pleurothallidinae) in Peru, including two new species. Anales del Jardín Botánico de Madrid 75 (1): e069. https://doi.org/10.3989/ajbm.2449.

Title in Spanish: Una sinopsis taxonómica de Acianthera (Orchidaceae: Pleurothallidinae) en Perú que incluye dos nuevas especies.

Received: 23-VI-2016; accepted: 28-II-2018; published on-line: 12-IV-2018; Associate Editor: E. Ortúñez.

\section{INTRODUCTION}

Acianthera Scheidw. - Orchidaceae Juss. - is a genus of the subtribe Pleurothallidinae Lindl. ex G.Don that consists of approximately 118-220 species (Pridgeon \& Chase 2001; Chiron \& van den Berg 2012; Chase \& al. 2015; Barros \& al. 2016; WCSP 2016). Its distribution covers tropical regions from the Antilles and Mexico to Argentina and Uruguay, being Brazil its main centre of biodiversity (Pridgeon 2005). Members of Acianthera are generally humid-forest or cloud-forest epiphytes from near sea level to over $2600 \mathrm{~m}$ a.s.l. and comprises species with more-or-less fleshy flowers, with connivent sepals, the lateral ones connate, lip articulated with the column, which is winged, and two pollinia (Pridgeon 2005; Luer 1986).

Almost two centuries have passed since Michael Joseph Scheidweiler described Acianthera in 1842. After Lindley (1859), species from this group were treated as Pleurothallis R.Br. for many decades, keeping the genus
Acianthera under the shade for nomenclatural purposes. Luer (1986) restored the name Acianthera as a subgenus of Pleurothallis in an attempt to accommodate species characterized by slightly fleshy flowers with connate lateral sepals borne singly or in a raceme from the base of the sessile leaf. Nevertheless, recent phylogenetic studies based on DNA (Pridgeon \& Chase 2001; Pridgeon \& al. 2001; Chiron \& van den Berg 2012; Chiron \& al. 2012; Karremans \& al. 2016) provided strong support for the recognition of Acianthera as a monophyletic group and the necessity to rank it to the generic level.

In Peru, 35 species of Acianthera have been recorded and treated hitherto under Pleurothallis (Schweinfurth 1958-1959; Luer 1986, 2004; Brako \& Zarucchi 1993; Bennett \& Christenson 1993-2001; Ulloa Ulloa \& al. 2004; León \& al. 2006) (figs. 1, 2). Most of the species occuring in the country are distributed on the northern slope of the Andes — Regions of Huánuco, San Martín, 

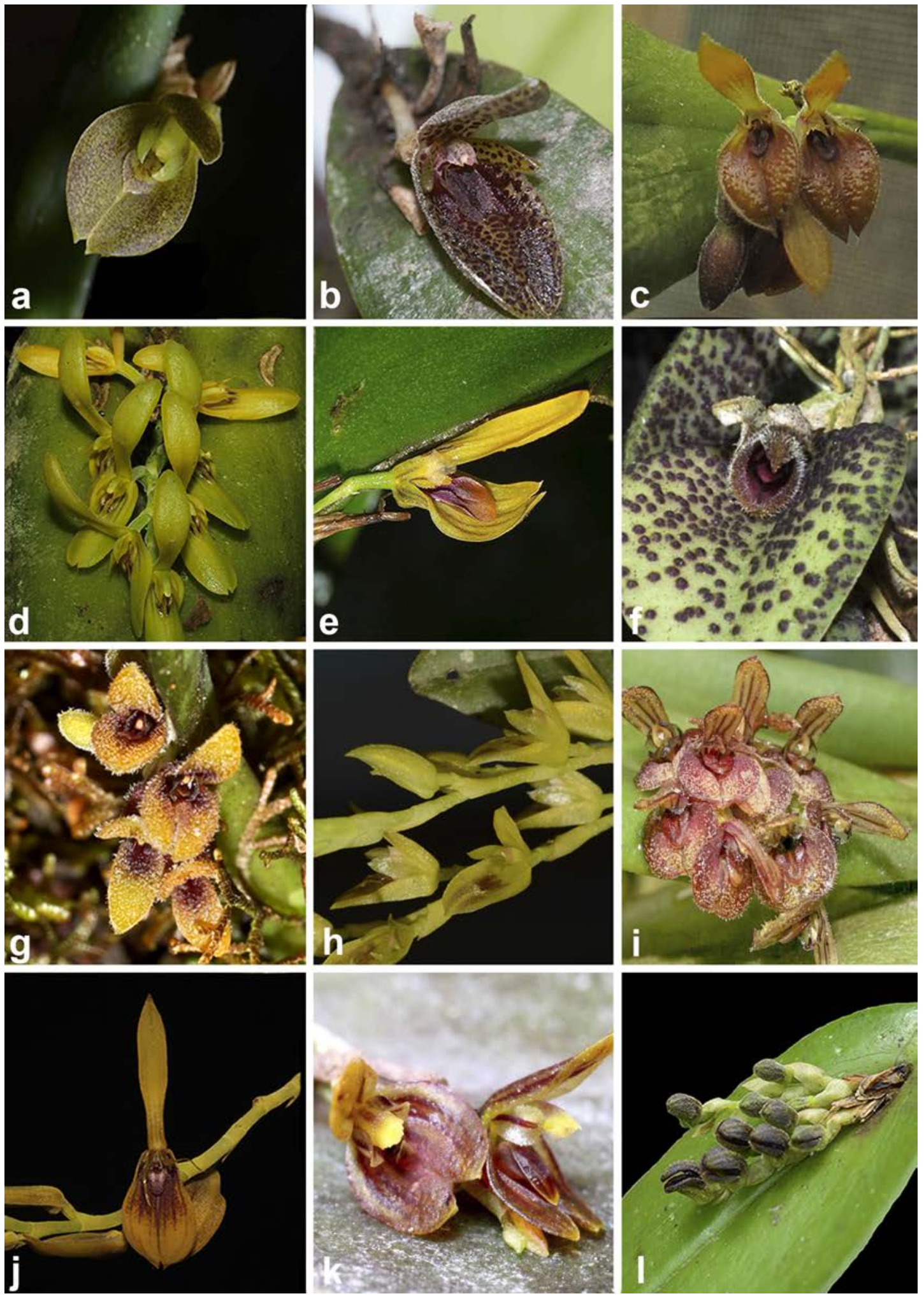

Fig. 1. Representative species of Peruvian Acianthera: a, A. berlinensis Damian \& al., sp nov.; b, A. biseta Luer; c, A. consatae (Luer \& R.Vásquez) Luer; d, A. decurrens (Poepp. \& Endl.) Pridgeon \& M.W.Chase; e, A. discophylla (Luer \& Carnevali) Luer; f, A. dodsonii (Luer) Karremans \& Rincón-Gonzales; $\mathbf{g}$, A. ericae Luer; $\mathbf{h}$, A. floribunda (Lindl.) F.Barros; i, A. lamia (Luer) Pridgeon \& M.W.Chase; j, A. lanceana (Lodd.) Pridgeon \& M.W.Chase; k, A. lojae (Schltr.) Luer; l, A. marleniae Damian \& al., sp nov. [Photographs: a, h, l, A. Damian; b, f, g, E. Moron; c, S. Olortegui; d, j, L. Ocupa; e, J. Janovec; i, J. Edquen; k, S. Arevalo.] 

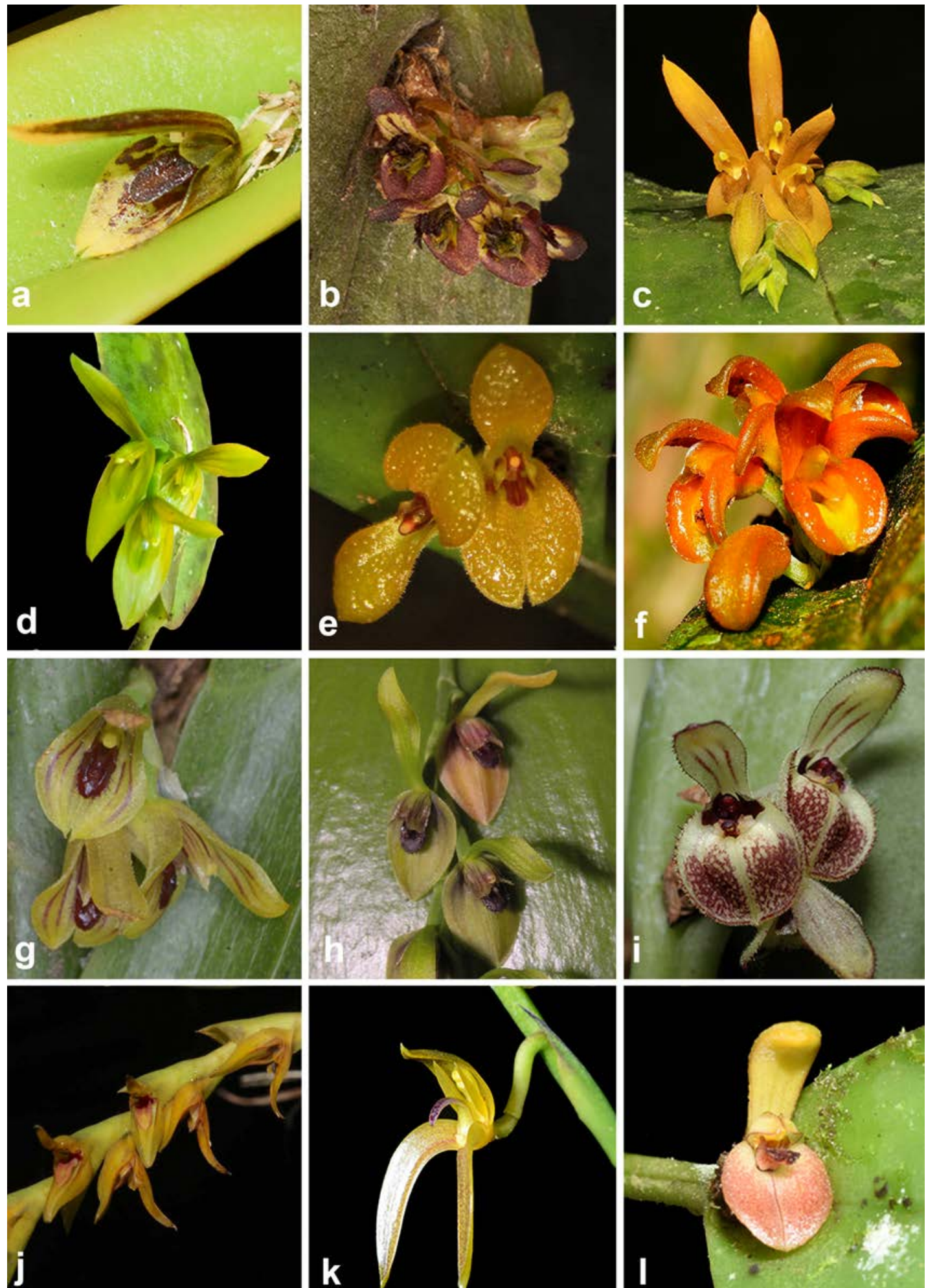

Fig. 2. Representative species of Peruvian Acianthera: a, A. miqueliana (H.Focke) Pridgeon \& M.W.Chase; b, A. pantasmoides (C.Schweinf.) Pridgeon \& M.W.Chase; c, A. polystachya (Ruiz \& Pav.) Pupulin; d, A. rodolfo-vasquezii Damian; e, A. rubroviridis (Lindl.) Pridgeon \& M.W.Chase; f, A. scalpricaulis (Luer) Pridgeon \& M.W.Chase; g, A. sicaria (Lindl.) Pridgeon \& M.W.Chase; h, A. toachica (Luer \& Dodson) Luer; i, A. tokachii (Luer) Luer; j, A. tricarinata (Poepp. \& Endl.) Pridgeon \& M.W.Chase; k, A. tunguraguae (F.Lehm. \& Kraenzl.) A.Doucette; l, A. wageneriana (Klotzsch) Pridgeon \& M.W.Chase. [Photographs: a, L. Torres; b, S. Arevalo; c, L. Egoavil; d, S. Olortegui; f, J. Edquen; g, A. Goicochea; e, j, K. Yupanqui; h, J. Janovec; i, E. Hunt; k, A. Damian; 1, L. Rimarachin.] 
Cajamarca, Amazonas - , where they grow as epiphytes in humid montane forests up to $3000 \mathrm{~m}$ a.s.1. According to León \& al. (2006), only one species belonging to Acianthera - Pleurothallis mexiae Luer; A. mexiae (Luer) Pridgeon \& M.W.Chase - was registered as endemic to Peru. However, the present study recognizes eight endemic species, including two new taxa described herein, and a few other taxa recently described (Damián 2018; Luer 2011; Luer \& Thoerle 2012). A taxonomic synopsis, an artificial key, and a map of all known localities for Peruvian Acianthera are provided by the authors.

\section{MATERIAL AND METHODS}

For taxonomic treatment, type information was acquired from the protologues of each cited name with the help of Biodiversity Heritage Library -www.biodiversitylibrary.org - and Botanicus -www.botanicus.org-. The type specimens were observed from online photos available through AMES, BM, BR, F, FLAS, K, NY, S, SP, P, US and W-abreviations of the herbaria according to Thiers (2017).

Each species was listed with its complete nomenclature information, including synonyms, original publication and type references. When the holotype was confirmed as destroyed, it was necessary to designate a lectotype. In some cases, we were unable to locate the holotype specimen cited by the protologue, or to be certain about its existence, in such cases we decided to cite as «unknown» rather than proposing a lectotype in order to avoid confusion, since we don't have the certainty of its real status - e.g., Pleurothallis wolfiana Schltr.

Specimens were obtained by revising Peruvian herbaria, USM, HOXA, MOL and CUZ, the latter from online photos available at Atrium - http://atrium.andesamazon.org/Through online databases from the AMES, BM, BR, F, FLAS, K, NY, S, SP, P, US and W, localities for all records were georeferenced and superimposed into a map of Peru using Qgis v. 2.18.0 software.

New species were photographed in situ using a Nikon D7100/Micro Nikkor $60 \mathrm{~mm}$ camera. The holotypes were deposited at UFV. Measurements and description of the morphological features were carried out using a Euromex SB-1903 stereomicroscope. For illustration of the new taxa, alcohol-preserved material was used as well as digital photos that were processed with Adobe Photoshop CC v. 14.0 .

\section{RESULTS AND DISCUSSION}

\section{Key to the species of Acianthera in Peru}

1. Lateral sepals free or barely connate at the base ........ 2

- Lateral sepals deeply connate
2. Sepals verrucose, dorsal sepal oblong-obovate .......... 2. A. aphthosa

- Sepals not verrucose, dorsal sepal triangular or oblong-triangular 3

3. Leaves erect, mostly more than $8 \mathrm{~cm}$ tall; sepals glabrous; petals with a lobule on the margin of both sides at the base 33. A. tunguraguae

- Leaves prostrate to pendent, mostly less than $5 \mathrm{~cm}$ tall; sepals pubescent externally; petals without lobules at the base 9. A. dodsonii

4. Inflorescence racemes 2-flowered or with a single flower

- Inflorescence racemes with more than two flowers ... 14

5. Lateral sepals deeply connate into a synsepal ............. 6

- Lateral sepals shallowly connate, usually separating easily 11

6. Sepals densely to shortly pubescent-ciliate .......... 7

- Sepals glabrous ...................................................... 8

7. Synsepal deeply convex; petals obovate; lip broadly rounded and shortly apiculate, densely verrucose at the apex 27. A. ruizii

- Synsepal slightly concave; petals elliptical; lip minutely erose, not verrucose at the apex ....... 4. A. biseta

8. Plants long repent ................. 28. A. saundersiana

- Plants caespitose or short repent .......................... 9

9. Leaves orbicular, round at the base; dorsal sepal narrowly linear .................... 11. A. erythrogramma

- Leaves elliptical to ovate, cuneate at the base; dorsal sepal oblong to oblanceolate ................................ 10

10. Petals acuminate with a longitudinal carina; column winged; lip entire ................ 25. A. rodolfo-vasquezii

- Petals acute without a longitudinal carina; column not winged; lip 3-lobed ................. 3. A. berlinensis

11. Leaves broadly elliptical to round; lateral sepals apiculate at the apex .................... 8. A. discophylla

- Leaves elliptical, narrowly linear to ovate; lateral sepals not apiculate at the apex ............................. 12

12. Leaves elliptical; apex of the lip usually coarsely verrucose, lateral lobes antrorse ..... 35. A. wageneriana

- Leaves linear to ovate; apex of the lip smooth to minutely verrucose, lateral lobes erect to retrorse .................. 13

13. Dorsal sepal much longer than the synsepal; petals minutely serrate in the margins above the middle ... 21. A. miqueliana

- Dorsal sepal not significantly longer than the synsepal; petals microscopically irregular in the margins ..................................... 12. A. fernandezii

14. Lip with a transverse basal callus articulated with the apex of the column-foot ......... 13. A. floribunda

- Lip hinged to the apex of the column-foot .......... 15

15. Lip trilobed or subtrilobed ................................ 16

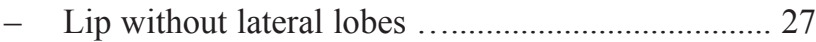

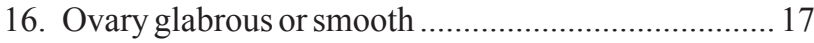

- Ovary pubescent ....................................................... 18 
17. Lip with apical lobe minutely serrulate, verrucose; lateral lobes near the middle, uncinate, antrorse

30. A. sicaria

- Lip with apical lobe microscopically to coarsely fringed, smooth; lateral lobes below the middle, broadly rounded, not antrorse ....... 23. A. polystachya

18. Leaves round to broadly elliptical ... 1. A. agathophylla

- Leaves narrowly elliptical to ovate 19

19. Lateral lobes with antrorse margins 20

- Laterallobes with rounded or sub-quadratemargins ...21

20. Petals oblong, acute, with a carina within each margin 5. A. carinata

- Petals flabellate-obovate, unguiculate, withouth carina 24. A. pubescens

21. Ramicauls triquetrous; petals unguiculate, acute at the apex 14. A. fumioi

- Ramicauls terete or compressed; petals obtuse to subacute at the apex ......................................... 22

22. Dorsal sepal apiculate ............................................. 23

- Dorsal sepal obtuse ................................................ 24

23. Ramicauls sharply 3 -winged, gradually broader toward the leaf; petals spathulate; lip subtrilobed

29. A. scalpricaulis

- Ramicauls not winged, more or less compressed and sulcate above; petals elliptical-oblong; lip trilobed .....

6. A. consatae

24. Lateral sepals deeply deflexed above the base; petals obovate, shortly acuminate ........... 32. A. tokachii

- Lateral sepals not deflexed above the base; petals oblong, elliptical to ovate, not acuminate 25

25. Lip densely verrucose with erose margins

19. A marleniae

- Lip not verrucose with minutely serrulate margins ... 26

26. Lip with a pair of tall, well-demarcated carinae 16. A. lamia

- Lip without well-demarcated carinae 22. A. pantasmoides

27. Inflorescence borne on top of the center of the leaf; dorsal sepals with the margin recurved ..... 7. A. decurrens

- Inflorescence borne at the base of the leaf; dorsal sepals with the margins not recurved ............................. 28

28. Ramicauls triquetrous or compressed .................... 29

- Ramicauls terete, sometimes compressed above ... 31

29. Leaves round at the base .................. 20. A. mexiae

- Leaves cuneate at the base ...................................... 30

30. Petals spathulate, obtuse; lip narrowly oblong, with a small lobule at each corner .............. 10. A. ericae

- Petals obovate, subacute; lip ovoid, without lobules .... .. 18. A. lojae

31. Petals apiculate to acuminate at the apex ......... 32

- Petals obtuse to subtruncate at the apex ............. 33

32. Petals serrate, obtuse, apiculate; lip broadly bilobed at the base ........................... 15. A. geminicaulina

- Petals fimbriate, acute, acuminate; lip minutely lobed at the base
33. Sepals pubescent, tuberculate within; synsepal obovate ..................................... 26. A. rubroviridis

- Sepals glabrous; synsepal oblong to ovate ............. 34

34. Inflorescences exceeding the leaf length; sepals thickly carinate, synsepal oblong ............. 33. A. tricarinata

- Inflorescences not exceeding the leaf lenght; sepals smooth, synsepal ovate 31. A. toachica

\section{Taxonomic Treatment}

1. A. agathophylla (Rchb.f.) Pridgeon \& M.W.Chase, Lindleyana 16 (4): 241 (2001); Pleurothallis agathophylla Rchb. f., Xenia Orchidacea 3: 25 (1881); Humboltia agathophylla (Rchb. f.) Kuntze, Revis. Gen. P1. 2: 667 (1891). Type: Bolivia, La Paz, Sorata, 2600 m a.s.1., Mar. 1860, Mandon 1132 (holo-: W; iso-: AMES!, K!, G!, S!, $\mathrm{P} !)$.

Pleurothallis cyclophylla Luer, Selbyana 3: 92 (1976). Type: Bolivia, Santa Cruz, near Samaipata, 1500 m a.s.1., 20 Oct. 1976, F. Fuchs s.n., [fl. in cult.], Luer 249 S (holo-: SEL!).

Pleurothallis nakatae T.Hashim., Ann. Tsukuba Bot. Gard. 4: 3 (1986). Type: Peru, Junín, Chanchamayo, near La Merced, 1700-1800 m a.s.l., 8 May 1985, Nakata 626 [fl. in cult.] (holo-: TNS, not located).

Distribution.-Bolivia and Peru (fig. 3).

Studied specimens.-PERU. Huánuco: south of Tingo Maria, Luer 5892 (SEL). Junín: Chanchamayo, near La Merced, Nakata 626 (TNS). Loreto: s.loc., Moore 10050 (Schweinfurth 1970).

2. A. aphthosa (Lindl.) Pridgeon \& M.W.Chase, Lindleyana 16 (4): 242 (2001); Pleurothallis aphthosa Lindl., Edwards's Bot. Reg. 24 (Misc.): $42-43$ (1838); Specklinia aphthosa (Lindl.) F.Barros, Hoehnea 10: 109 (1984). Type: s.loc., Jun. 1838, Bateman s.n. [received from Mr. Bateman, who in turn received it from the Birmingham Botanical Garden, without a name] (holo-: K!).

Pleurothallis macrophyta Barb.Rodr., Gen. Spec. Orchid. 1: 19 (1877). Type: Brasil, Minas Gerais, Pico de Frade near Caldas, Barbosa s.n. (holo-: B, lost; lecto-, here designated: Sprunger 1996: 211 tab. 153B).

Pleurothallis pelioxantha Barb.Rodr., Gen. Spec. Orchid. 1: 17 (1877). Type: Brasil, Minas Gerais, Carmo do Rio Claro, Barbosa s.n. (holo-: B, lost; lecto-, here designated: Sprunger (1996: 230 tab. 172).

Pleurothallis ciliata var. abbreviata C.Schweinf., Bot. Mus. Leafl. 16: 46 (1953). Type: Peru, Huánuco, Yanano, ca. $6000 \mathrm{ft}$ a.s.l., 13 May 1923, Macbride 3837 (holo-: AMES!).

Distribution.-Colombia, Ecuador, Peru (fig. 3), Bolivia and Brazil.

Studied specimens.-PERU. Junín: Chanchamayo, above hacienda santa blanca, S. B-3856 (MO).

3. A. berlinensis Damian, Chiron, Mitidieri \& Rimarachin, sp. nov. Type: Peru, Amazonas, Bagua, Bosque Berlin, disturbed area, 1900 m a.s.1., 9 Dec. 2015, A. Damián and L. Rimarachin 01166 (holo-: UFV!). Figs. 1a, 4, 5.

\section{LSID: urn:1sid:ipni.org:names:77176586-1}

Similar to Acianthera biseta Luer from which it differs by the leaf shorter than the ramicaul and proportionally wider, a shorter peduncle bearing 2 flowers, smaller, glabrous flowers, with yellowish sepals, an 
oblong and 3-veined dorsal sepal, acute petals with slightly erose margins above the middle, and a narrower lip with obtuse apex with a mucro.

Plant epiphytic, caespitose, $24 \mathrm{~cm}$ tall. Roots $1 \mathrm{~mm}$ in diameter, slender, densely fasciculate. Ramicaul stout, erect, terete, $7-14 \mathrm{~cm}$ long, enclosed by 2 tubular sheaths near its base. Leaf erect, thickly coriaceous, sessile, elliptical, obtuse, $8-9.5 \mathrm{~cm}$ long, 2-2.7 $\mathrm{cm}$ wide, the base cuneate, sessile. Inflorescence 1-3 successively 2-flowered racemes, peduncle c. $1 \mathrm{~cm}$ long, subtended by a spathaceous bract, ovate $0.5-1 \mathrm{~cm}$ long from the base of the leaf; floral bracts infundibular, thin, obtuse, $5 \mathrm{~mm}$ long; ovary $4 \mathrm{~mm}$ long; sepals fleshy, rigid, microscopically papillate on the margins, yellowish externally, densely spotted with dark purple on the adaxial surface, the dorsal sepal oblong, subacute, $9 \mathrm{~mm}$ long, 2-2.5 $\mathrm{mm}$ wide, 3 -veined, the lateral sepals connate up to near their apices into an ovate synsepal, obtuse, shortly bilobed, 6-veined, $8 \mathrm{~mm}$ long, $5 \mathrm{~mm}$ wide, each lobe shortly apiculate, the base forming a shallow mentum with the column foot; petals fleshy, yellow, with 3 red rows along the veins, oblong-oblanceolate, somewhat falcate, acute, with margins slightly erose above the middle, thickened at the apex, 3-veined, $5 \mathrm{~mm}$ long, $1 \mathrm{~mm}$ wide; lip yellowish, covered densely with dark purple dots, fleshy, glabrous, trilobed, oblong-oblanceolate, obtuse, shortly apiculate, $4 \mathrm{~mm}$ long, $1.5 \mathrm{~mm}$ wide, serrate along the apical margin, the lateral lobes near the middle, erect, uncinate, acute, antrorse, the disc with a pair of smooth low, parallel carinae descending from the lateral lobes and extending toward the apex, the base truncate, thick, shortly concave, hinged to the apex of the column-foot. Column semiterete, $4 \mathrm{~mm}$ long, with ventrally extended wings, clinandrium completely covering the anther and denticulate at the margin, the foot thick, $1 \mathrm{~mm}$ long, stigma ventral, anther, pollinia and capsule not seen.

Distribution.-Known only from the type locality in the province of Bagua —Amazonas - within «Bosque Berlin» Conservation Area (fig. 3). It grows as an epiphyte on low branches of remnant trees of the cloud forest, in semi-shady conditions.

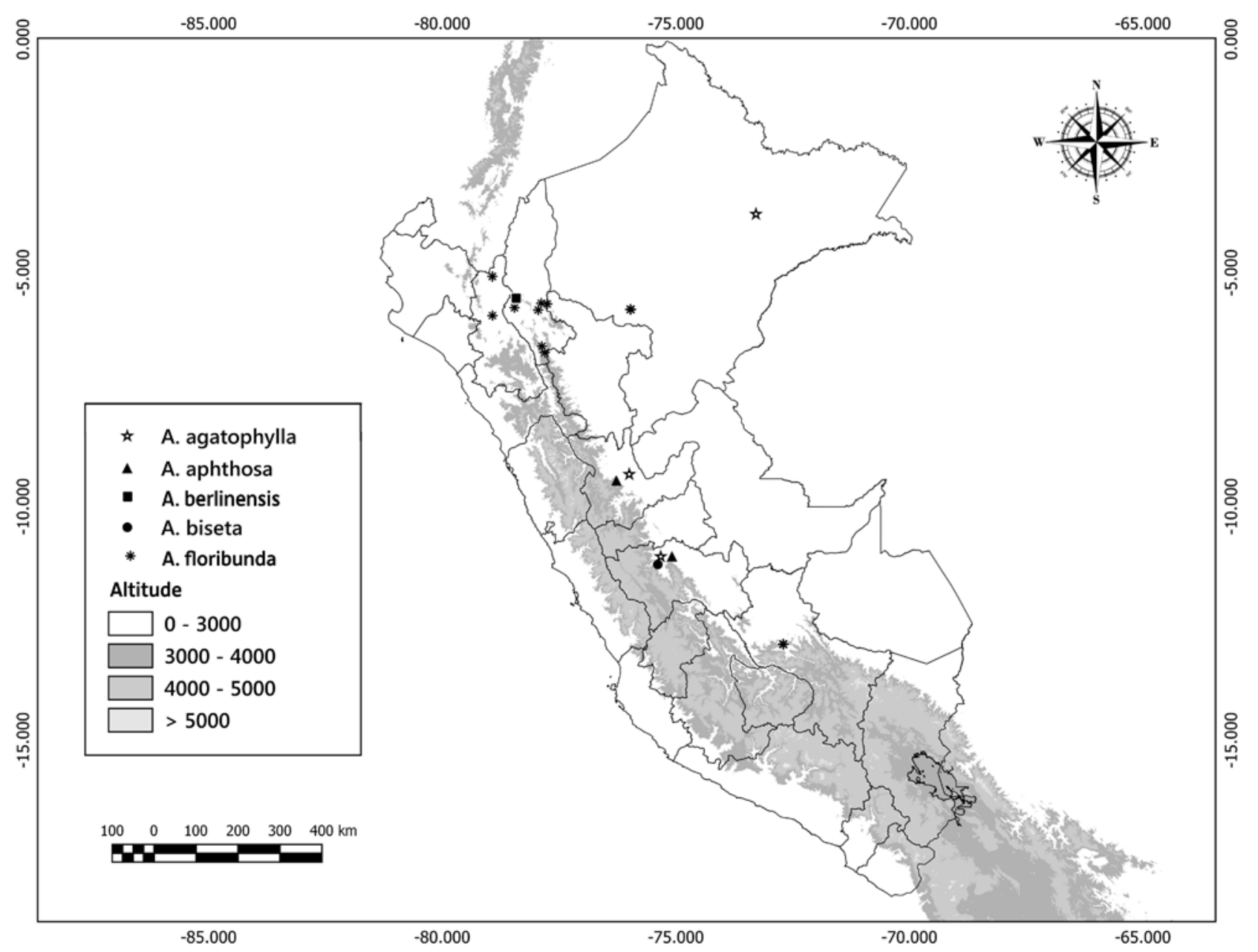

Fig. 3. Map showing the known Peruvian localities for Acianthera agathophylla (Rchb. f.) Pridgeon \& M.W.Chase, A. aphthosa (Lindl.) Pridgeon \& M.W.Chase, A. berlinensis Damian \& al., sp. nov., A. biseta Luer and A. floribunda (Lindl.) F.Barros. 

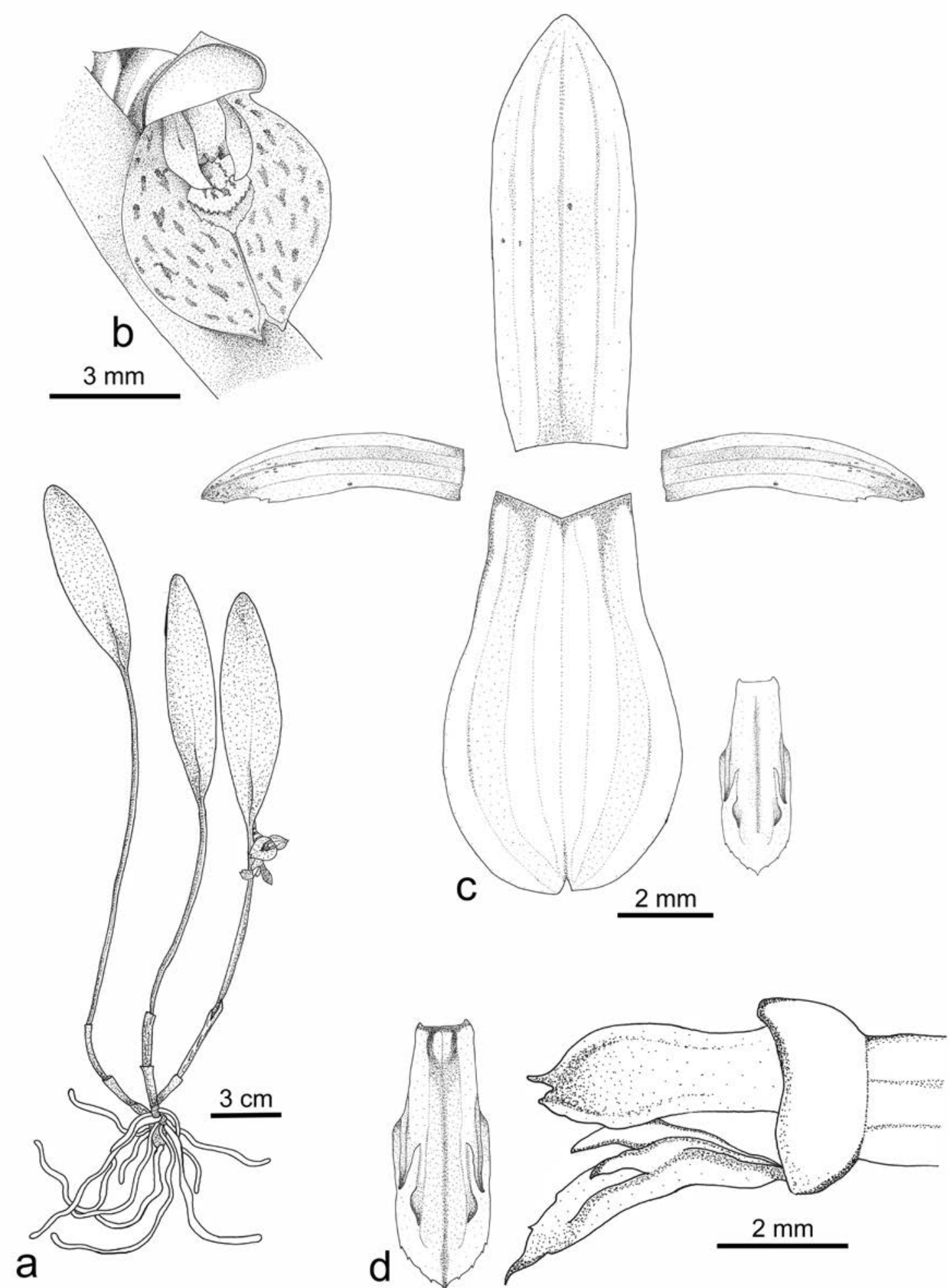

Fig. 4. Acianthera berlinensis Damian, Chiron, Mitidieri \& Rimarachin, sp. nov.: a, habit; b, flower; c, dissected perianth; d, column and lip, lateral view, and lip in frontal view. [A. Damián and L. Rimarachin 1166 (holo-: UFV); drawn by G. Damián P.] 


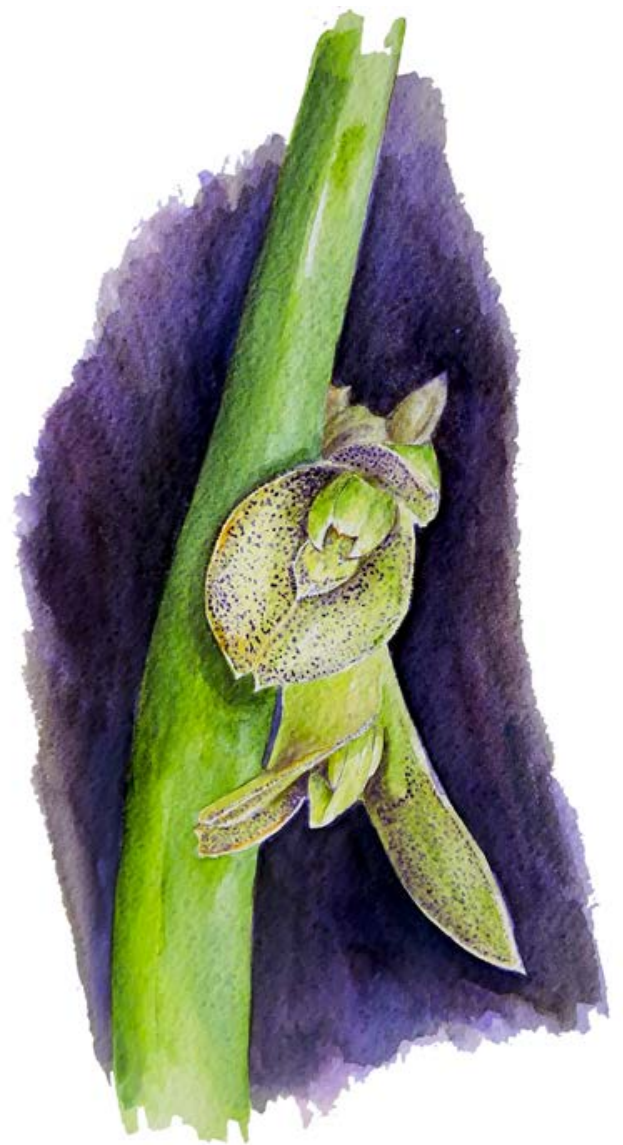

Fig. 5. Acianthera berlinensis Damian, Chiron, Mitidieri \& Rimarachin, sp. nov. [A. Damián and L. Rimarachin 1166 (holo-: UFV); watercolour by Ó. Vilca Pinares.]

Etymology.-In honor to ACP-Bosque Berlin, where Leyda Rimarachin and her family make great efforts to preserve a beautiful forest on the high mountains of Bagua - Amazonas-Peru.

Comments.-This species is somewhat similar to A. biseta Luer, a recently described Peruvian species. As the latter, it belongs to the group of Acianthera characterized by stout, erect, terete ramicauls, thickly coriaceous elliptical leaves and a very short inflorescence bearing 1-2 flowers. However it differs from $A$. biseta by its wider leaves shorter than the ramicauls — vs. equal to the ramicauls-, its 2-flowered inflorescence - vs. 1-flowered-, its smaller flowers - c. $9 \mathrm{~mm}$ long versus $16 \mathrm{~mm}$-yellowish with a lip densely dark purple dotted — vs. dull greenish with a deep purple lip), its glabrous sepals — vs. densely and shortly pubescent-, its oblong, 3-veined dorsal sepal —vs. elliptical, 5-veined-, its acute petals with erose margins - vs. obtuse and marginally entire - , its narrower lip with an obtuse mid-lobe — vs. rounded-, without horseshoe-shaped callus at the base.

Another similar species is A. auriculata (Lindl.) Pridgeon \& M.W.Chase, wich differs from $A$. berlinensis on having long inflorescences, almost equal to the leaves bearing 5 or more flowers - vs. short inflorescence bearing 1-2 flowers-, flowers small, almost half the dimensions of $A$. berlinensis — sepals, $5 \mathrm{~mm}$ vs. $9 \mathrm{~mm}$ long; synsepal, 2.5-3 mm vs. $5 \mathrm{~mm}$ wide- , carinate sepals and transverse callus at the base of the lip — vs. non carinate sepals and lack of the transverse callus.

4. A. biseta Luer, Harvard Pap. Bot. 17: 333, fig. 1 (2012). Type: Peru, Junín, Chanchamayo, near Mina San Vicente,
2000-2800 m a.s.1., Jun. 2012, B. Monterrey s.n. [Morón 004] (holo-: USM, not located). Fig. 1 b.

Distribution.-Endemic to Peru, known only from the type locality (fig. 3).

5. A. carinata (C.Schweinf.) Luer, Monogr. Syst. Bot. Missouri Bot. Gard. 95: 253 (2004); Pleurothallis carinata C.Schweinf., Botanical Museum Leaflets 15: 85 (1951). Type: Peru, Cusco, Quispanchis, Maurayacu, Marcapata, 1300-160 m a.s.1., 12 Dec. 1943, Vargas 3757 (holo-: AMES!).

Distribution. - Endemic to Peru (fig. 6).

Studied specimens._PERU. Apurimac: Abancay, Vargas 8977 (CUZ).

6. A. consatae (Luer \& R.Vásquez) Luer, Monogr. Syst. Bot. Missouri Bot. Gard. 95: 253 (2004); Pleurothallis consatae Luer \& R.Vásquez, Revista Soc. Boliv. Bot. 2 (2): 135 (1999). Type: Bolivia, La Paz, Prov. Muñecas, near consata, 21 Jan. 1981, Luer et al. 6323 (holo-: SEL). Fig. 1c.

Distribution.-Bolivia and Peru (fig. 6). It was reported for Huánuco by Blas (2015: 49) as Acianthera sp.

Studied specimens.-PERU. San Martín: Rioja, Elias Soplin Vargas, Santa Fe, Olortegui 001 (UFV!).

7. A. decurrens (Poepp. \& Endl.) Pridgeon \& M.W.Chase, Lindleyana 16 (4): 243 (2001); Pleurothallis decurrens Poepp. \& Endl., Nov. Gen. Sp. Pl. 1: 48, pl. 83 (1836); Humboltia decurrens (Poepp. \& Endl.) Kuntze, Revis. Gen. P1. 2: 667 (1891). Type: Peru, Huánuco, ad praedium Cuchero, Dec. 1829, Poeppig 1604 (holo-: W!). Fig. 1d.

Distribution.-Ecuador and Peru (fig. 6).

Comments.-This eccentric species varies in color among different populations in Peru, from yellow densely suffused with dark purple sepals - more common forma-, through entirely yellow flowers (fig. 1d).

Studied specimens.-PERU. Amazonas: Bongara, Río Utcubamba. Pomacocha, Hutchison and Wright 3970 (MO); Bongara, km 320 (Olmos-Rioja), Hutchison and Wright 6841 (USM!, MO); Chachapoyas, between Ingenio and Pomacochas, Lopez et al. 4282 (AMES, HUT); Bagua, La Peca, «Bagua chica», Ocupa s.n. (USM-photo!); Chachapoyas, Leimebamba, Salas s.n. (USM-photo!). Huánuco: Muña, Macbride 40555 (F); «Palanda», Andre 4632 (K); Río Huallaga below Río Santo Domingo, Macbride 4252 (AMES, F!). Junín: Huacapistana, Killip and Smith 24221 (USM!, AMES, US); Junín (Jauja), Hajeck 99 (USM!); Chanchamayo, Hajek 80 (USM!). Pasco: Oxapampa, Chontabamba, west of Oxapampa, low pass between Chontabamba and Suiza, Foster et al. $6731 A(\mathrm{MO})$.

8. A. discophylla (Luer \& Carnevali) Luer, Monogr. Syst. Bot. Missouri Bot. Gard. 95: 253 (2004); Pleurothallis discophylla Luer \& Carnevali, Novon 3 (2): 158, fig. 1 (1993). Type: Ecuador, Morona-Santiago, epiphytic in tall forest along Río Upano $\mathrm{N}$ of Macas, $1100 \mathrm{~m}$ a.s.1., 15 Jan. 1989, Luer et al. 13927 (holo-: MO!). Fig. 1e.

Distribution.-Bolivia, Ecuador, Peru and Venezuela (fig. 6). 
Studied specimens.-PERU. Amazonas: Condorcanqui, Río Cenepa, vicinity of Huampami, Kujikat 94 (MO). Cusco: Quispicanchis, 600 $1700 \mathrm{~m}$ a.s.1., Chambi et al. 666 (BRIT!). Loreto: Mishana, Río Nanay, Solomon 3540 (MO); Maynas, Iquitos, Revilla 4310 (MO); Maynas, Campamento no 1, Mishana, Rio Nanay, Ruiz 258 (MO); Maynas, Dtto. Iquitos, Allpahuayo, Estación experimental del instituto de investigaciones de la Amazonia Peruana (IIAP), Vásquez and Jaramillo 16628 (MO); Maynas, Mishana, Vásquez et al. 5305 (MO); Maynas, Allpahuayo, Iquitos, Nauta, Vásquez and Criollo D. 5773 (MO); Maynas, Iquitos, Santa María de Nanay, Gentry et al. 36521 (MO); Maynas, Dtto. Iquitos, km 8 Carretera Quisto Cocha, McDaniel and Rimachi 27836 (MO); Maynas, Río Nanay, Caserío Mishana, Foster 4436 (USM!, MO, F!); Maynas, Dtto. Iquitos, carretera de Iquitos a Nauta, Rimachi Y. 7457 (MO, USM!); Requena, Dtto. Saquena, Río Ucayali, Rimachi Y. 3700 (MO); Requena, Vásquez and Jaramillo 4890 (MO). Puno: Río Tavara, Gentry et al. 76893 (USM!, MO). San Martín: Maristal Caceres, Dtto. Tocache Nuevo, Puerto Pizana, Schunke 6891 (MO).

9. A. dodsonii (Luer) Karremans \& Rincón-Gonzales, Phytotaxa 238 (2): 174-182 (2015); Pleurothallis dodsonii Luer, Selbyana 3 (1,2): 96 (1976); Apoda-prorepentia dodsonii (Luer) Luer, Monogr. Syst. Bot. Missouri Bot. Gard. 95: 255 (2004). Type: Ecuador, Tungurahua, epiphitic near Río Blanco east of Baños, 1600 m a.s.1., 8 Oct. 1961, Dodson and Thien 889 (holo-: SEL!). Fig. 1f.

Distribution.-Ecuador and Peru (fig. 6).

Studied specimens.-PERU. Amazonas: Bagua, Bosque Berlín, A. Damián and L. Rimarachin 1168 (UFV!).

10. A. ericae Luer, Harvard Pap. Bot. 17: 333, fig. 1 (2012). Type: Peru, Junín,Chanchamayo, near Mina San Vicente, 2000-2800 m a.s.1., Jun. 2012, B. Monterrey s.nl [Morón 003] (holo-: USM, not located). Fig. $1 \mathrm{~g}$.

Distribution.-Endemic to Peru, known only from the type locality (fig. 7).

11. A. erythrogramma (Luer \& Carnevali) Luer, Monogr. Syst. Bot. Missouri Bot. Gard. 95: 253 (2004); Pleurothallis erythrogramma Luer \& Carnevali, Novon 3 (2): 160, fig. 2 (1993). Type: Ecuador, Napo, epiphytic on trees along Río Cascales E of Limbaqui, 450 m a.s.1., 9 Feb. 1986, Luer et al. 11766 (holo-: MO!).

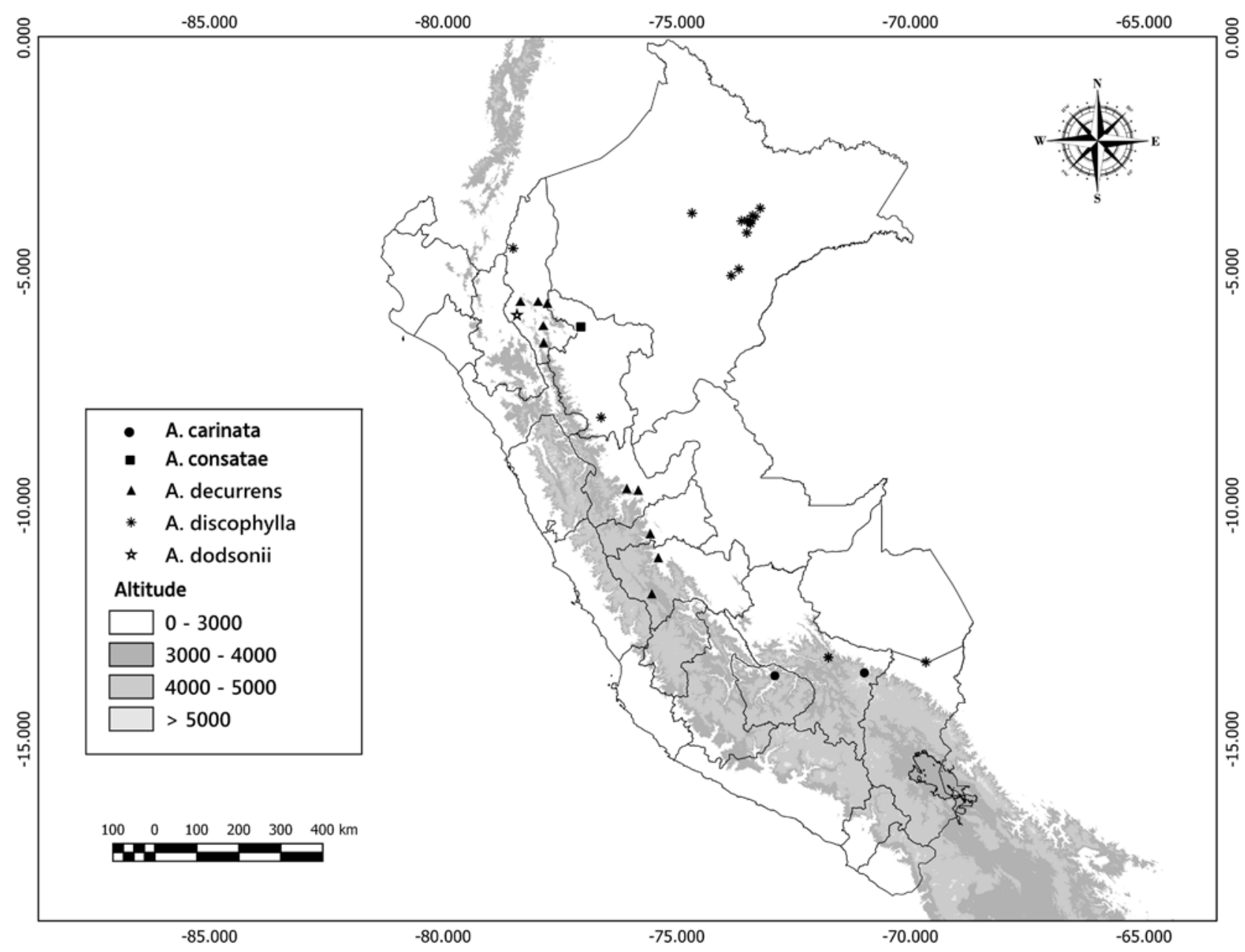

Fig. 6. Map showing the known Peruvian localities for Acianthera carinata (C.Schweinf.) Luer, A. consatae (Luer \& R.Vásquez) Luer, A. decurrens (Poepp. \& Endl.) Pridgeon \& M.W.Chase, A. discophylla (Luer \& Carnevali) Luer and A. dodsonii (Luer) Karremans \& Rincón-Gonzales. 
Distribution.-Ecuador and Peru (fig. 7).

Studied specimens.-PERU. Amazonas: S of Aintami, E of Cenepa, Berlin 1605 (MO). Pasco: Oxapampa, Dtto Palcazu, Parque Nacional Yanachaga Chemillen, Estación Biológica Paujil, Ureta et al. 052 (USM!); Dtto. Palcazu, Comunidad Nativa Yanesha Loma Linda, $411 \mathrm{~m}$ a.s.l., Vásquez et al. 36659 (HOXA!); Dtto. Palcazu, Reserva Comunal Yanesha-Sector Azulis, 670 m a.s.1., Vásquez et al. 29638 (HOXA!); Cacazu, $1200 \mathrm{~m}$ a.s.1., Hajeck 331 (USM!).

12. A. fernandezii Luer, Harvard Pap. Bot. 16 (2): 313, 2 (2011). Type: Peru, Loreto, Maynas, Puerto Almendra, Río Nanay, 104 m a.s.1., 13 Aug. 2008, Fernández and Ruiz 3513 (holo-: USM, not located).

Distribution.-Endemic to Peru (fig. 7).

13. A. floribunda (Lindl.) F.Barros, Bradea 8: 294 (2002); Specklinia floribunda Lind1., Gen. Sp. Orchid. P1.: 9 (1830); Dendrobium ophioglossoides Sieber ex Lindl., Gen. Sp. Orchid. P1.: 9 (1830), pro syn.; Specklinia ophioglossoides Mutel, Mém. Soc. Roy. Centr. Agric. Dépt. N. 1835-1836: 84 (1837), nom. illegit. superfl.; Pleurothallis floribunda
(Lindl.) Lindl., Edwards’s Bot. Reg. 28 (Misc.): 73 (1842), nom. illegit., non Poepp. \& Endl., Nov. Gen. Sp. P1. 1: 48-49, tab. 84 (1835) [1836]; Pleurothallis sieberi Luer, Monogr. Syst. Bot. Missouri Bot. Gard. 20: 34 (1986); Acianthera sieberi (Luer) Pridgeon \& M.W.Chase, Lindleyana 16: 246 (2001); Acianthera floribunda (Lindl.) F.Barros, Bradea 8: 294 (2002); Arthrosia floribunda (Lindl.) Luer, Monogr. Syst. Bot. Missouri Bot. Gard. 105: 248 (2006). Type: Martinique, Sieber 206 (holo-: K; iso-: M!, P!, MO!). Fig. 1h.

Pleurothallis capillaris Lindl., Edwards's Bot. Reg. 21: sub tab. 1797 (1836); Humboltia capillaris (Lindl.) Kuntze, Revis. Gen. Pl. 2: 667 (1891); A. capillaris (Lindl.) Pridgeon \& M.W.Chase, Lindleyana 16 (4): 242 (2001); Specklinia capillaris (Lindl.) Luer, Monogr. Syst. Bot. Missouri Bot. Gard. 95: 259 (2004); Arthrosia capillaris (Lindl.) Campacci, Bol. CAOB 69-70: 26 (2008). Type: Brazil, Sebastianopolit., Martius s.n. (holo-: M!)

Pleurothallis longicaulis Lindl., Edwards's Bot. Reg. 28 (Misc.): 72 (1842); Humboltia longicaulis (Lindl.) Kuntze, Revis. Gen. Pl. 2: 667 (1891); Acianthera longicaulis (Lindl.) Pridgeon \& M.W.Chase, Lindleyana 16: 244 (2001); Specklinia longicaulis (Lindl.) Luer, Monogr. Syst. Bot. Missouri Bot. Gard. 95: 261 (2004); Arthrosia longicaulis

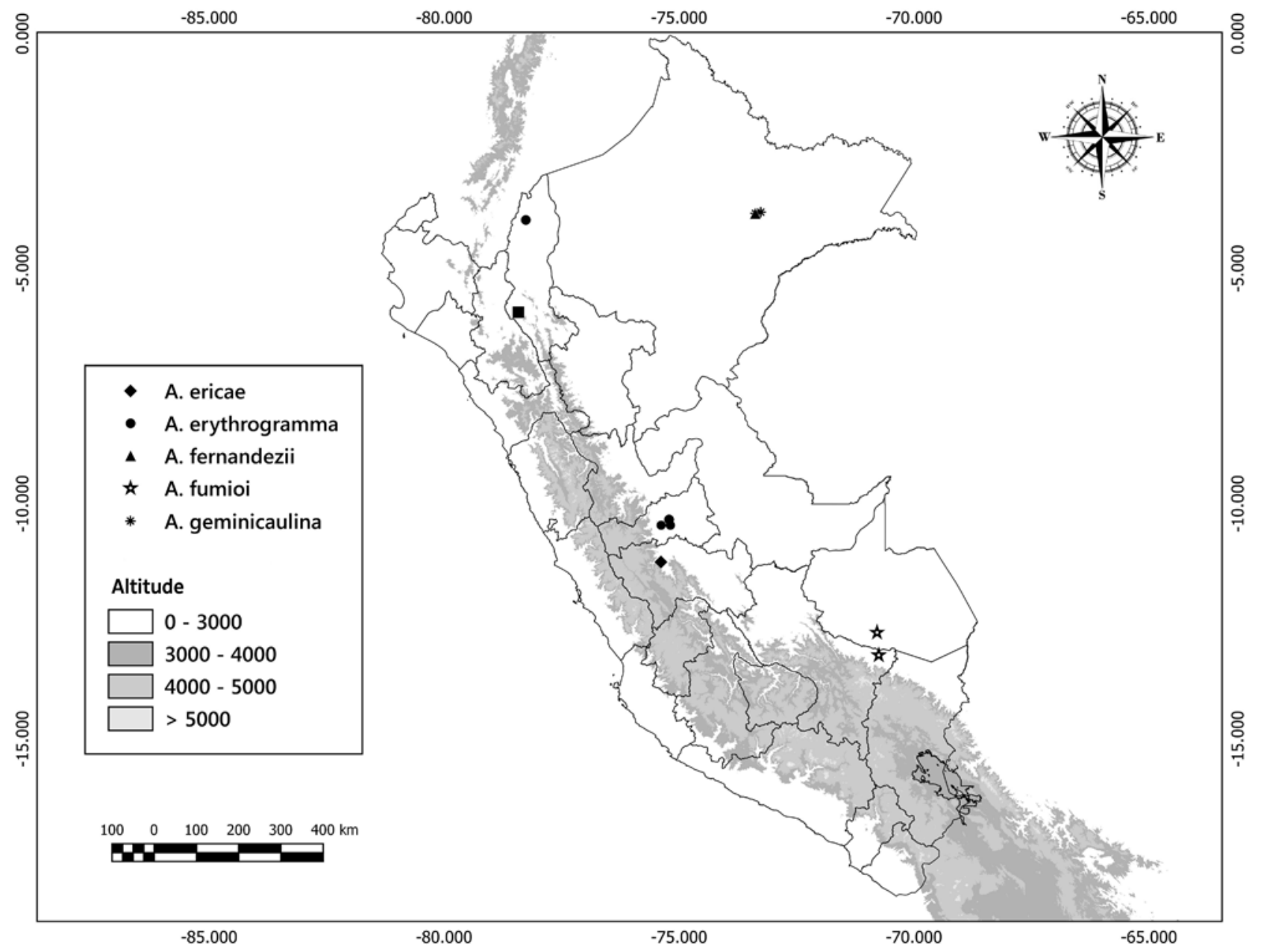

Fig. 7. Map showing the known Peruvian localities for Acianthera ericae Luer, A. erythrogramma (Luer \& Carnevali) Luer, A. fernandezii Luer, A. fumioi (T.Hashim.) Luer and A. geminicaulina (Ames) Pridgeon \& M.W.Chase. 
(Lindl.) Campacci, Bol. CAOB 69-70: 27 (2008). Type: Brazil, Rio de Janeiro, Serra dos Orgãos, s.coll. (holo-: BM!).

Pleurothallis triquetra Schltr., Repert. Spec. Nov. Regni Veg. 12: 488 (1913), nom. illegit., non Klotzsch ex Lindl., Fol. Orchid. Pleurothallis: 13 (1859). Type: Bolivia, Tres Cruces, ca. 1500 m a.s.1., Feb. 1911, Herzog 1627 (holo-: B, destroyed).

Distribution.-Brazil, Ecuador, Peru and West Indies (fig. 3).

Comments.-Lindley described this species in 1830 as Specklinia floribunda including Dendrobium ophioglossoides as a synonym, based on a specimen obtained by Sieber from Martinique. Later in 1832, Lindley himself transfered Specklinia floribunda to Pleurothallis (Pleurothallis floribunda) without noticing that Poeppig and Endlicher already published that name on 1836 on his Nova Genera ac Species Plantarum, therefore Pleurothallis floribunda (Lind1.) Lindl. became an illegitimate name. Luer (1986), on his treatment of the genus Pleurothallis, presented the subgenus Arthrosia to accommodate nine species, including Pleurothallis longicaulis and a new name for Specklinia floribunda (as Pleurothallis sieberi), listing Pleurothallis floribunda (Lindl.) Lindl. nom. illegit. and Pleurothallis ophiglossoides sensu Garay \& Sweet as synonyms. Based on phylogenetic studies, Pridgeon \& Chase (2001), supported the monophyly of Acianthera, and ranked the subg. Acianthera of Luer to the generic level, transfering several epithets to it, including A. sieberi, A. capillaris and A. longicaulis and reduced several subgenus proposed by Luer (1986) to synonyms including subg. Arthrosia. Later, Luer (2002) reinstated several species to Pleurothallis and reduced epithets transfered by Pridgeon \& Chase (2001) to synonyms. Luer accepted Pleurothallis capillaris and listed Pleurothallis longicaulis, Pleurothallis sieberi, A. sieberi, A. capillaris and A. longicaulis as synonyms. Luer (2004) recognized the generic rank of Acianthera without including any reference to its neglected subg. Arthrosia or any of its members. Meanwhile, Barros (2002) proposed a new combination for Specklinia floribunda (A. floribunda) here recognized as the accepted name for this species, with priority over Pleurothallis capillaris (1836). Afterward, Luer (2006) elevated Arthrosia to the generic rank and proposed several transfers, including a new combination to Specklinia floribunda (Arthrosia floribunda). Chiron \& van den Berg (2012) on its revision of Acianthera reduced the genus Arthrosia to A. sect. Arthrosiae (Luer) Chiron \& van den Berg, including A. capillaris as one of its members. Finally, Karremans \& al. (2016) recognized a broad concept of Acianthera, which includes four infrageneric ranks, reducing Arthrosia and $A$. sect. Arthrosiae into synonyms of $A$. subg. Acianthera.

Acianthera floribunda is characterized by erect ramicauls, caespitose; oblong, obtuse leaves; lanceolate sepals, laterals carinate; spathulate to lanceolate with denticulate margins petals; 3-lobed oblong, acute labellum with two parallel longitudinal lamella, a conspicuously transverse callus across the base; and terete with lacerate apex column - features taken from the protologue of A. floribunda and Luer (2009).

Studied specimens.-PERU. Cajamarca: San Ignacio, Coordillera del Condor, Calatayud and Vásquez 520 (MO); San Ignacio, Cordillera del Condor, Calatayud et al. 534 (MO); San Ignacio, Cordillera del Condor, Calatayud et al. 535 (MO, CUZ!, USM!); San Ignacio, Calatayud and Vargas, 6856 ${ }^{a}, 6848$ (USM!). Cusco: Calca, Yanatile, Quebrada Honda, Damián 8031 (UFV!). Amazonas: Suyobamba, $3 \mathrm{~km}$ NE of Pedro Ruiz Gallo, Gentry et al. 61306 (MO); Chachapoyas, Bennett 3436 (MO); Between Pomacochas and Moyobamba, Tokach P-64 (MO); Prov. of Bongara, Rio Utcubamba, $300 \mathrm{~km}$ east of Olmos, Hutchison and Wright 6848 (F, USM!); Vicinity of Campamiento ingenio, Hutchison and Wright 3840 (USM!); Prov. of Bagua, between Bagua Grande and Chachapoyas, Ferreyra 14425 (USM!); Prov. Bongara, Campamento Ingenio on Rio Utcubamba, Hutchison and Wright $6856^{\circ}$ (USM!). San Martín: Yurimaguas, Hajek 2196 (USM!)

14. A. fumioi (T.Hashim.) Luer, Monogr. Syst. Bot. Missouri Bot. Gard. 95: 253 (2004); Pleurothallis fumioi T.Hashim., Jap. J. Bot. 46: 173 (1971). Type: Peru, Puno, between Quince Mil and Puente Rio Inambari, Hashimoto and Wada s.n. (holo-: TI).

Pleurothallis phoxophylla Luer, Revista Soc. Boliv. Bot. 3: 57 (2001). Type: Peru, Puno, Carabaya, Puente Inambari toward Madre de Dios, 520 m a.s.1., 8 Dec. 1966, Vargas 18426 (holo-: AMES!).

Distribution.-Endemic to Peru (fig. 7). WCSP (2016) erroneously reported this species as endemic to Bolivia.

Studied specimens.-PERU. Madre de Dios: Madre de Dios, Tambopata, C. Vargas 18425 (CUZ).

15. A. geminicaulina (Ames) Pridgeon \& M.W.Chase, Lindleyana 16 (4): 243 (2001); Pleurothallis geminicaulina Ames, Schedul. Orchid. 6: 59-60 (1923). Type: Costa Rica, La Unión, Turrialba, 3500 ft a.s.1., 12 May 1923, Lankester 454 (holo-: AMES!).

Distribution.-Costa Rica, Ecuador, Panama and Peru (fig. 7).

Studied specimens.-PERU. Loreto, Maynas, Iquitos, Vásquez and Jaramillo 8702 (MO); Maynas, Puerto Almendras, Croat 18537 (MO).

16. A. lamia (Luer) Pridgeon \& M.W.Chase, Lindleyana 16 (4): 244 (2001); Pleurothallis lamia Luer, Selbyana 5 (2): 170 (1979). Type: Ecuador, Azuay, 3000 m a.s.1., 18 Jul. 1977, Luer et al. 1744 (holo-: SEL!). Fig. 1i.

Distribution.-Ecuador and Peru (fig. 8).

Studied specimens.-PERU. Amazonas, Luya, Leymebamba, 2950 m a.s.1., Salas 128 (USM!).

17. A. lanceana (Lodd.) Pridgeon \& M.W.Chase, Lindleyana 16 (4): 244 (2001); Pleurothallis lanceana Lodd., Bot. Cab. 18 (177): pl. 1767 (1832); Humboltia lanceana (Lodd.) Kuntze, Rev. Gen. Pl. 2: 667 (1891). Type: Suriname, Jun. 1831, Lance s.n. (holo-: lost; lecto-: t.1767, K 000079847!, here designated). Fig. 1j.

Pleurothallis ciliata Knowles \& Westc., Floral Cabinet 1: 39-40, tab. 19 (1837); Humboltia ciliata (Knowles \& Westc.) Kuntze, Rev. Gen. Pl. 2: 667 (1891); A. ciliata (Knowles \& Westc.) F.Barros \& L.R.S.Guim., Neodiversity 5: 28 (2010). Type: Guyana, Demerara, near the Falls of Ouripano on the Masseroni River, 1834, Henchman s.n. (holo-: not seen).

Pleurothallis plumosa Lindl., Edwards's Bot. Reg. 28 (Misc.): 72 (1842); Humboltia plumosa (Lindl.) Kuntze, Rev. Gen. P1. 2: 668 (1891). Type: Trinidad, s.d., Loddiges 13 (holo-: K).

Pleurothallis crassifolia H.Focke, Tijdschr. Wis-Natuurk. Wetensch. Eerste KI. Kon. Ned. Inst. Wetensch. 2: 196-197 (1849), nom. illegit. superfl., non Rchb.f., Linnaea 22: 832 (1849) [1850]; Humboltia crassifolia (H.Focke) Kuntze, Rev. Gen. Pl. 2: 667 (1891). Type: Surinam, near Para River, s.d., Focke s.n. (holo-: illustr. at W, not found; iso-: BR!).

Pleurothallis minax Rchb. f., Bonplandia 2 (2): 24 (1854). Type: Venezuela, Caracas, Wagener 562 (holo-: W; iso-: BR!).

Pleurothallis serrifera Lindl., Folia Orchidaceae 9: 34 (1859); Humboltia serrifera (Lindl.) Kuntze, Rev. Gen. Pl. 2: 668 (1891). Type: Brazil, Amazonas, Caatingas near Panuré, Spruce 2724 (holo-: K; iso-: P!).

Pleurothallis sprucei Lindl., Folia Orchidaceae 9: 35 (1859); Humboltia sprucei (Lindl.) Kuntze, Rev. Gen. Pl. 2: 668 (1891). Type: Brazil, Amazonas, «low sandy woods called Caatingas by the river Uaupés», Spruce 2725 (holo-: K; iso-: P!). 
Pleurothallis daguensis F.Lehm. \& Kraenzl., Bot. Jahrb. Syst. 26 (3-4): 444 (1899). Type: Colombia, Sucre, on Dagua River, Lehmann 8167 (holo-: B, destroyed).

Pleurothallis huebneri Schltr., Beih. Bot. Centralbl. 42 (2): 90-91 (1925). Type: Brazil, Amazonas, Taracuá, Alto Rio Negro, Hübner 173 (holo-: B, destroyed).

Pleurothallis ciliata var. elongata C.Schweinf., Bot. Mus. Leafl. 16 (3): 47-48 (1953). Type: Peru, Loreto, vicinity of Iquitos, Dec. 1936, Klug 10013 (holo-: AMES!).

Distribution.-Bolivia, Colombia, Costa Rica, Ecuador, French Guiana, Guyana, Panama, Peru (fig. 8), Suriname and Venezuela.

Comments.-According to Barros \& Guimarães (2010), the correct name for this taxon is A. ciliata (Westc.) F.Barros \& L.R.S.Guim., based on Pleurothallis ciliata Westc. published in 1837. However, in the present work we decided to accept Pleurothallis lanceana Lodd. as the right basionym for this taxon, since it was first published in 1832 by Loddiges in his Botanical Cabinet and not in 1859 by Lindley as stated by WCSP (2016). Consequently, the valid name is A. lanceana in accordance with McNeill \& al. (2012)

Studied specimens.-PERU. Amazonas: Condorcanqui, Río Cenepa, Vásquez et al. 19020 (MO); Bagua, District Imaza, right bank of stream, Shimutaz, Díaz 7655 (MO). Cusco: Paucartambo, road from Patrias to the North, Maas et al. 6154 (USM!, MO). Huánuco: Leoncio prado, near Tingo María, Jara s.n. (MO); Leoncio Prado, Dtto. Rupa Rupa, northeast of Tingo María, near Mapresa, Schunke V. 10631 (MO). Huancavelica: Tajacaya, Huachocolpa, 2300 m a.s.1., Leon et al. 2992 (MOL!). Madre de Dios: Tambopata, Madre de Dios river, northern margin, Householder et al. 769 (BRIT!). Loreto: vicinity of Iquitos, Klug 10050 (AMES); Quebrada Cuninico, Croat 17792 (MO); Itaya river above Iquitos, Croat 19221 (MO); Tacsha Curaray river, of Río Napo, Croat 20402 (MO); Alto Amazonas, orillas del Río Pastaza, entre Rimachi y Río Witoyacu, Díaz S. et al. 1319 (MO); Maynas, Iquitos, Revilla 3590 (MO); Maynas, Rio Yavari, Gentry and Revilla 20861 (USM!, MO); Maynas, Yanamono, Explorama Tourist Camp, halfway between Indiana and mouth of Rio Napo, van der Werff et al. 9920 (MO); Maynas, Dtto. Indiana, Quebrada de Yanayacu, McDaniel and Rimachi 26585 (MO); Loreto (Maynas), carretera Iquitos-Nauta, zona reservada, Alpahuayo Mishana, Weng 48 (USM!); Dtto. Nauta, Caserio Miraflores, $132 \mathrm{~m}$ a.s.l., Vásquez et al. 34764 (HOXA!). Pasco: Oxapampa, Palcazu valley, Rio San Jose in the Rio Chuchurras, Smith 4010 (MO); Palcazu, Comunidad Nativa San Pedro de Pichanaz, Sector Azulis, Cercanias del Centro Comunal, Monteagudo et al. 10168 (USM!, HOXA!, MO, HUT, AMAZ, MOL!); Dtto Pozuzo, Fundo Agustin Egg Schuller, $840 \mathrm{~m}$ a.s.1., Becerra et al. 1393 (HOXA!); Dtto Palcazu, Comunidad San Pedro de Pichanaz, Sector Francisco, 832 m a.s.1., Valenzuela and Heredia 12216 (HOXA!); Dtto Palcazu, Reserva Comunal Yanesha, Comunidad Nativa 7 de Junio, 410 m a.s.1., Monteagudo et al. 11522 (HOXA!); Dtto. Palcazu, Centro Connas, 373 m a.s.1., Vásquez et al. 36508 (HOXA!). San Martín: Marical Caceres, Toache nuevo, Quebrada de mantención, Shunke 7781 (AMES,

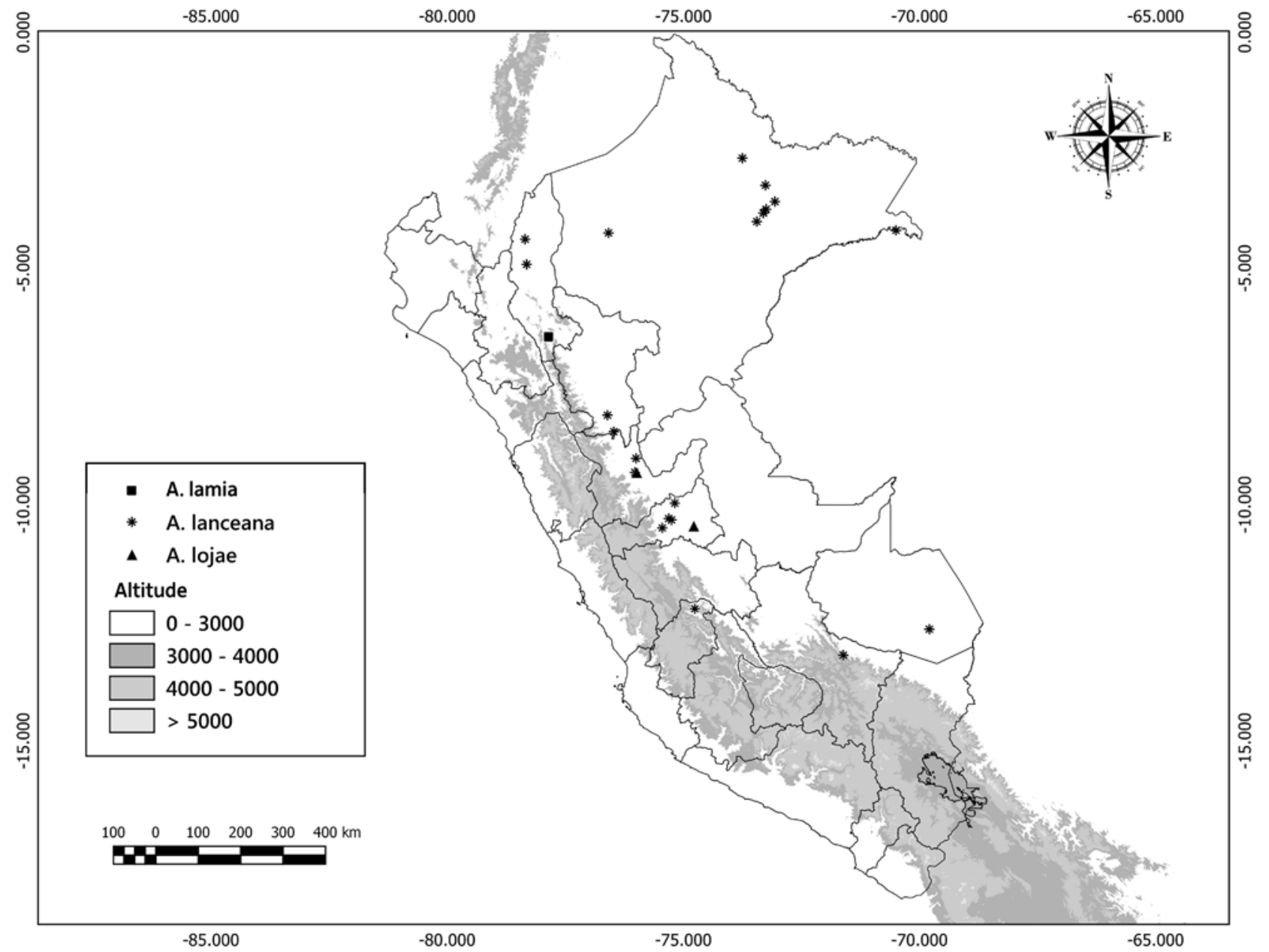

Fig. 8. Map showing the known Peruvian localities for Acianthera lamia (Luer) Pridgeon \& M.W.Chase, A. lanceana (Lodd.) Pridgeon \& M.W.Chase and A. lojae (Schltr.) Luer. 
MO); Mariscal Caceres, Dtto. Uchiza, Cachiyacu de Lepuna, Schunke V. 7307 (MO).

18. A. lojae (Schltr.) Luer, Monogr. Syst. Bot. Missouri Bot. Gard. 95: 254 (2004); Pleurothallis lojae Schltr., Repert. Spec. Nov. Regni Veg. Beih. 8: 60-61 (1921). Type: Ecuador, Loja, on trees in dense forests of the eastern slopes of the eastern Andes of Loja, 1300-1600 m a.s.1., Lehman 8202 (holo-: K!). Fig. 1k.

Pleurothallis brunnescens Schltr., Repert. Spec. Nov. Regni Veg. Beih. 19: 183 (1923); A. brunnescens (Schltr.) Karremans, Harvard Pap. Bot 21 (2): 171-187 (2016). Type: Costa Rica, Alajuela, San Pedro de San Ramón, 1100 m a.s.1., Jun. 1921, Brenes 27 (holo-: B, destroyed). Costa Rica, Prov. Alajuela, San Isidro de San Ramón, 1075 m a.s.l., 12 Nov. 1923, Brenes 824 (neo-: CR, designated by Barringer 1986).

Pleurothallis citrophila Luer, Selbyana 3: 266 (1977); A. citrophila (Luer) Pridgeon \& M.W.Chase, Lindleyana 16: 243 (2001). Type: Panama, Coclé, above El Valle, 1000 m a.s.1., 2 Sep. 1976, Luer and Butcher 1375 (holo-: SEL!).

Pleurothallis florosa Luer, Selbyana 3: 302 (1977); A. florosa (Luer) Pridgeon \& M.W.Chase, Lindleyana 16: 243 (2001). Type: Ecuador, Manabí, s.loc., Luer 1573 (holo-: SEL!).

Distribution.-Bolivia, Costa Rica, Ecuador, Panama and Peru (fig. 8).

Comments.-Karremans \& al. (2016) proposed a new combination for Pleurothallis brunnescens (A. brunnescens) suggesting that it should be treated as an autonomous species. However, they based their decision on vegetative morphology and conjectures regarding the different flora between Ecuador and Costa Rica. After revising the holotypes of Pleurothallis lojae and Pleurothallis brunnescens, it states clear that both species represent the same concept with slightly variations, Pleurothallis brunnescens present oblong leaves (vs. elliptical) and acute lip (vs. obtuse to oblong).

Studied specimens.-PERU. Huánuco: Tingo María, Luer 2901 (SEL). Pasco: Oxapampa, 5 km SE of Oxapampa, Smith 3639 (USM!, MO).

19. A. marleniae Damian, Chiron \& Mitidieri, sp. nov. Type: Peru, Cajamarca, Chota, Querocoto, entrance road to «La Granja», $2600 \mathrm{~m}$ a.s.1., May 2014, A. Damián and N. Mitidieri 03090 (holo-: UFV!). Figs. 11, 9.

\section{LSID: urn:1sid:ipni.org:names:77176588-1}

Similar to Acianthera sicula (Luer \& Vásquez) Luer but differs in having larger flowers, carinate sepals, fimbriate sub-auriculate petals, and obovoid lip with erose, broadly subquadrate lateral lobes.

Plant small, epiphytic, roots unknown. Ramicauls incomplete, erect, more than $15 \mathrm{~cm}$ long, gradually becoming sharply winged above, $0.8-1 \mathrm{~cm}$ wide at the junction with the leaf. Leaf erect, coriaceous, narrowly elliptical-ovate, obtuse, 7-9 cm long, 2-4 cm wide, sessile, cuneate, decurrent on the stem for 1-2 cm. Inflorescence in a crowded raceme, with 4-6 distichous flowers reclining on the blade of the leaf, $2.3 \mathrm{~cm}$ long, from a spathe $1 \mathrm{~cm}$ long at the apex of the secondary stem imbedded well above the base of the leaf; floral bracts $0.4-0.6 \mathrm{~mm}$ long; pedicel $3.5 \mathrm{~mm}$ long; ovary $3 \mathrm{~mm}$ long, densely pubescent; sepals dark purple, densely pubescent on abaxial surface, glabrous within but verrucose in the upper third, the dorsal sepal oblong-obovate, obtuse, 5-6 $\mathrm{mm}$ long, $3 \mathrm{~mm}$ wide, connate to the lateral sepals for $0.75 \mathrm{~mm}$, the lateral sepals connate to near the apex into a thick, oblong, concave synsepal, carinate, $6 \mathrm{~mm}$ long, $4 \mathrm{~mm}$ wide, with the apex shortly bifid; petals purple, oblong, obtuse, $4 \mathrm{~mm}$ long, $2 \mathrm{~mm}$ wide, with the margins sharply fimbriate above the upper third, 1-veined, the base sub-auriculate; lip dark brown, thick, fleshy, obovoid, 3-lobed, $4 \mathrm{~mm}$ long, $2.5 \mathrm{~mm}$ wide, the apex broadly rounded, densely verrucose with erose margins, the lateral lobes erect below the middle, low, erose, broadly sub-quadrate, $1 \mathrm{~mm}$ long, the disc with a thick pair of parallel, verrucose carinae from the base to near the middle, the base hinged to the columnfoot between a pair of membranous auricles; column stout, semiterete, 3.5-4 mm long, with ventrally extended wings, clinandrium entirely covering the anther with lacerate margins, the foot thick, short, $1 \mathrm{~mm}$ long, stigma ventral, anther cap cucullate, obovate, capsule not seen.

Distribution.-Peru, in the mountains of Chota - Cajamarca- (fig. 10), where it grows as an epiphyte in montane forest at about $2600 \mathrm{~m}$ a.s.l.

Etymology.-In honor of the senior author's maternal family.

Comments. - Acianthera marleniae sp. nov. is most similar to A. sicula. Vegetatively, the latter differs by having ramicauls half wider at the apex $(5 \mathrm{~mm})$ compared to $A$. marleniae sp. nov. $-1 \mathrm{~cm}-$. Moreover, A. sicula presents cuneate leaves which are decurrent on the stem for less than $1 \mathrm{~cm}$ vs. $2 \mathrm{~cm}$ in $A$. marleniae sp. nov. Flowers of $A$. marleniae sp. nov. are distinguished from those of $A$. sicula by their larger size, carinate sepals and dark purple-green color — vs. brown, yellow brown-. Petals are quite similar in shape but larger $-4 \times 2 \mathrm{~mm}-$, sub-auriculate at the base and densely fimbriate in A. marleniae sp. nov. vs. shorter $-2.5 \times$ $1 \mathrm{~mm}-$, not auriculate and shortly denticulate in A. sicula. Instead of ovoid, ovate-obtuse lip with lateral lobes rounded in A. sicula, the lip of A. marleniae sp. nov. is obovoid with lateral lobes low, erose and broadly sub-quadrate.

Another similar species is $A$. wyvern (Luer \& R.Escobar) Pridgeon \& M.W.Chase, which differs from $A$. marleniae sp. nov. on having longer flowers — sepals—, $10 \times 3.5 \mathrm{~mm}$ — vs. $5 \times 3 \mathrm{~mm}$ - , obovate petals — vs. oblong — and oblong-obovate lip with oblique, oblong, lateral lobes —vs. obovoid, subquadrate lateral lobes.

20. A. mexiae (Luer) Pridgeon \& M.W.Chase, Lindleyana 16 (4): 244 (2001); Pleurothallis mexiae Luer, Monogr. Syst. Bot. Missouri Bot. Gard. 76: 174 (1999); Pleurothallis chamensis var. tenuis C.Schweinf., Bot. Mus. Leafl. 10: 176 (1942). Type: Peru, Huánuco, Churubamba, Hda. Mercedes, Cotirarda, $1560 \mathrm{~m}$ a.s.1., Mexia $8215^{a}$ (holo-: AMES!).

Distribution.-Endemic to Peru, known only from the type collection (fig. 10).

21. A. miqueliana (H.Focke) Pridgeon \& M.W.Chase, Lindleyana 16 (4): 244 (2001); Specklinia miqueliana H.Focke, Tijdschr. Wis-Natuurk. Wetensch. 2: 199-200 (1849); Pleurothallis miqueliana (H.Focke) Lindl., Fol. Orchid. 9: 17 (1859); Stelis miqueliana (H.Focke) Lindl., Fol. Orchid. 9: 17 (1859); Humboltia miqueliana (H.Focke) Kuntze, Revis. Gen. Pl. 2: 667 (1891). Type: Surinam, river of Para, on the trunks of Crescentia cujete, Apr. 1853, Focke s.n. (holo-: W; iso-: BR!). Fig. 2a. 

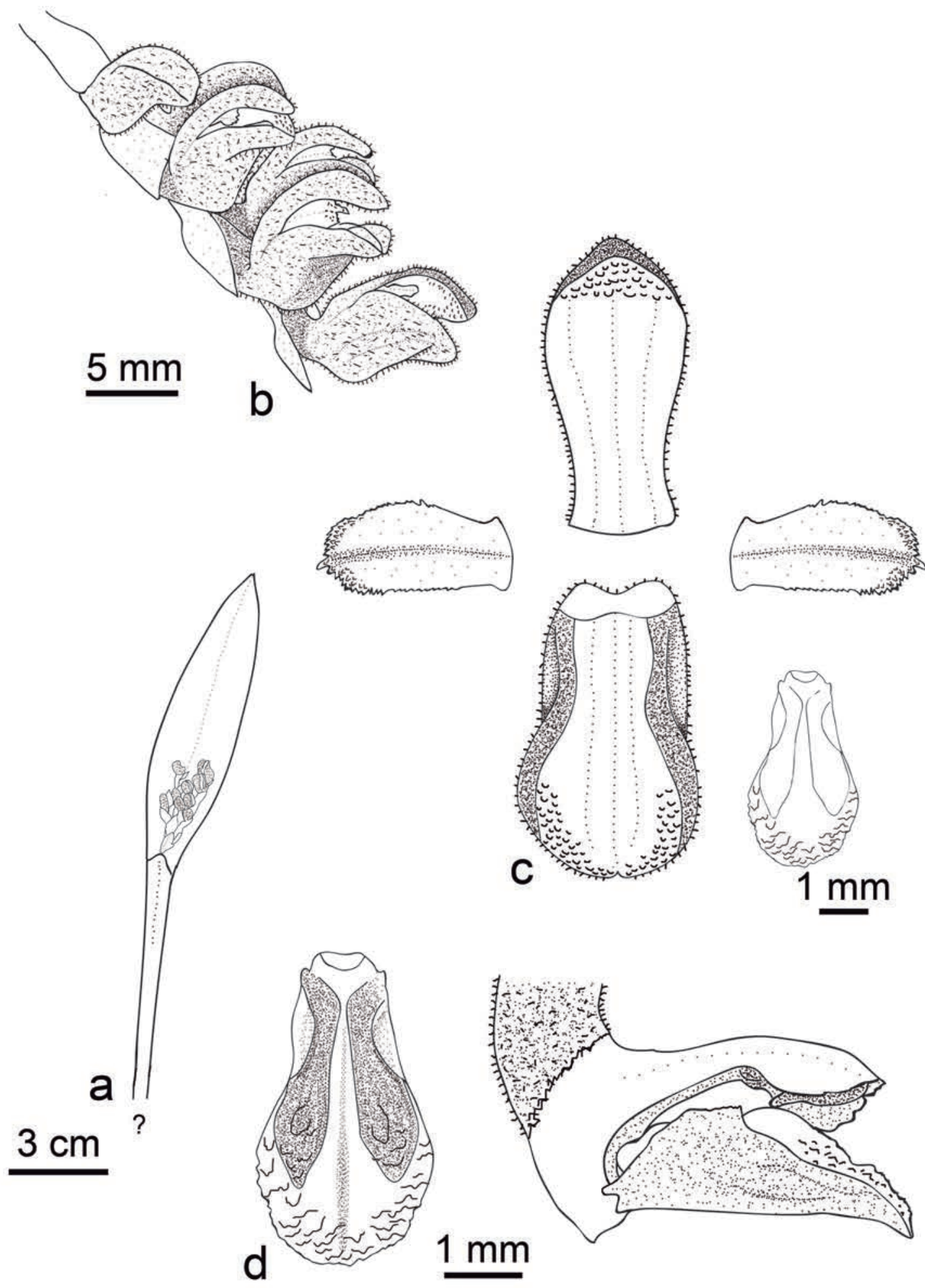

Fig. 9. Acianthera marleniae Damian, Chiron \& Mitidieri, sp. nov.: a, habit; b, flower; c, dissected perianth; d, column and lip, lateral view, and lip in frontal view. [A. Damián 03090 (holo-: UFV!); drawn by A. Damián.] 
Pleurothallis fimbriata Lindl., Fol. Orchid. 9: 17 (1859); Humboltia fimbriata (Lindl.) Kuntze, Revis. Gen. Pl. 2: 667 (1891). Type: Brazil, Panuré, Caatingas, Spruce 2459 (holo-: K!; iso-: BR!, P!).

Pleurothallis longisepala Barb.Rodr., Vellosia ed. 2, 1: 115 (1891) Type: Brazil, Amazonas, near Uatukurá, rio Yauapery, Barbosa s.n. (holo-: probably lost; lecto-, designated here: Sprunger 1996: 182, tab. 126A).

Distribution.-Brazil, Ecuador, Guyana, Suriname, Peru (fig. 9) and Venezuela.

Studied specimens.-PERU. Loreto, vicinity of Iquitos, Killip and Smith 26998 (AMES, US!); ibid., Klug 10126 (AMES); Gamitanaccha, Río Mazan, on the bank above the trees, Schunke 162 (AMES, K, US!, USM!).

22. A. pantasmoides (C.Schweinf.) Pridgeon \& M.W.Chase, Lindleyana 16 (4): 245 (2001); Pleurothallis pantasmoides C.Schweinf., Bot. Mus. Leafl. 15 (3): 98 (1951). Type: Peru, Cusco, Paucartambo, YanamayoTambomayo, 1800 m a.s.1., 4 May 1947, Vargas 6499 (holo-: AMES!). Fig. 2b.

Distribution.-Colombia, Ecuador and Peru (fig. 10).
Studied specimens.-PERU. Amazonas: Chachapoyas, Cerros Calla Calla, E Side, $5 \mathrm{~km}$ above Leimbembamba on the road to Balsas, at San Miguel, Hutchison and Bennett 4566 (MO). Cajamarca: Cutervo, Distrito San Andres de Cutervo, Parque Nacional de Cutervo, Diaz and Osores 2601 (USM!, MO). Cusco: Prov. of Paucartambo, YanamayoTambomayo, Vargas 6499 (AMES!); Paucartambo, Atalaya, Junction of Rio Carbon, Nuñez et al. 15545 (MO, CUZ!); Paucartambo, Moscoso 843 (MO); ibid., Hutchison 6959 (USM!). Huancavelica: Ampurco, between Salcabamba and Surcubamba, Tovar 3771 (USM!).

23. A. polystachya (Ruiz \& Pav.) Pupulin, Anales Jard. Bot. Madrid 69: 27 (2012); Humboltia polystachya Ruiz \& Pav., Syst. Veg. Fl. Peruv. Chil.: 234 (1798); Stelis polystachya (Ruiz \& Pav.) Willd., Sp. Pl. 4: 139 (1805). Type: Peru, «hábitat in montibus Chinchao supra arbores», Ruiz and Pavón s.n. (holo-: MA). Fig. 2c.

Pleurothallis casapensis Lindl., Edwards's Bot. Reg. 28 (Misc.): 76 (1842); Humboltia casapensis (Lindl.) Kuntze, Revis. Gen. P1. 2: 667 (1891); A. casapensis (Lindl.) Pridgeon \& M.W.Chase, Lindleyana 16: 242 (2001). Type: Peru, Huánuco, Cassapi, Mathews 1830 (holo-: K).

Pleurothallis chamensis Lindl., Orchid. Linden.: 2 (1846); Humboltia chamensis (Lindl.) Kuntze, Revis. Gen. P1. 2: 667 (1891); A. chamensis

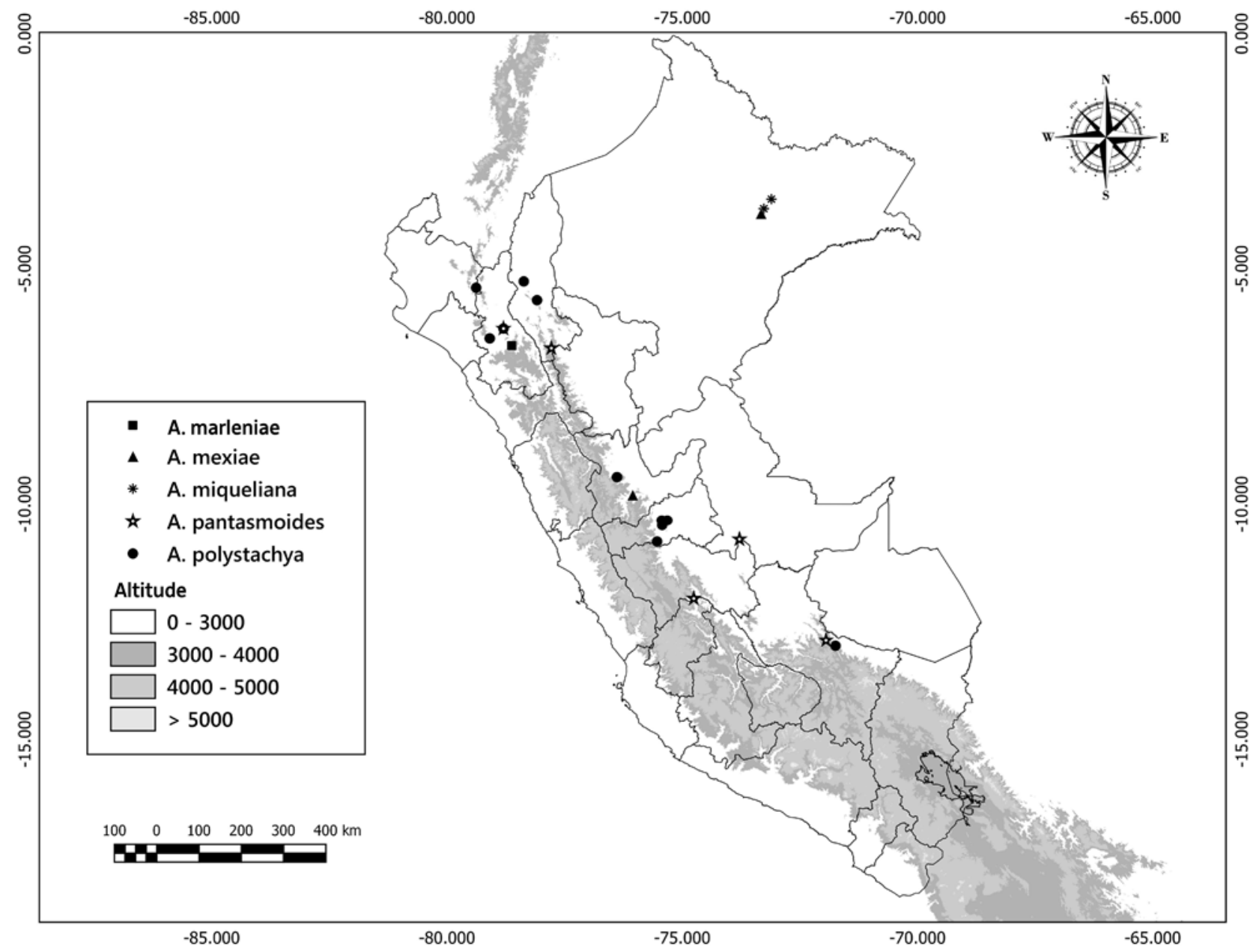

Fig. 10. Map showing the known Peruvian localities for Acianthera marleniae Damian \& al., sp. nov., A. mexiae (Luer) Pridgeon \& M.W.Chase, A. miqueliana (H.Focke) Pridgeon \& M.W.Chase, A. pantasmoides (C.Schweinf.) Pridgeon \& M.W.Chase and A. polystachya (Ruiz \& Pav.) Pupulin. 
(Lindl.) Pridgeon \& M.W.Chase, Lindleyana 16: 242 (2001). Type: Venezuela, Mérida, along Rio Chamá, $5000 \mathrm{ft}$ a.s.l., May 1842, Linden 639 (holo-: $\mathrm{K}$ !; iso-: $\mathrm{P}$ !).

Pleurothallis triangularis Klotzsch \& H.Karst., Allg. Gartenzeitung 15: 329 (1847). Type: Venezuela, Caracas, $6000 \mathrm{ft}$ a.s.1., Otto 911 (holo-: K; iso-: $\mathrm{W})$.

Pleurothallis triquetra Klotzsch ex Lindl., Fol. Orchid. 9: 13 (1859). Type: Venezuela, Dist. Fed., Karsten s.n. (holo-: K).

Pleurothallis phyllostachys Schltr., Repert. Spec. Nov. Regni Veg. Beih. 9: 76 (1921); A. phyllostachys (Schltr.) Pridgeon \& M.W.Chase, Lindleyana 16: 245 (2001), syn. nov. Type: Peru, Huacabamba, 2500 m a.s.l., Apr. 1912, Weberbauer 6313 (holo-: B, probably destroyed; lecto-, designated by Luer (2004): AMES!).

Pleurothallis wolfiana Schltr., Repert. Spec. Nov. Regni Veg. Beih. 8: 64 (1921). Type: Ecuador, Chimboirazo, near Puente de Chimbo, Sodiro s.n. (holo-: B, unknown).

Pleurothallis coffeicola Schltr., Repert. Spec. Nov. Regni Veg. 27: 50 (1929); A. coffeicola (Schltr.) Pridgeon \& M.W.Chase, Lindleyana 16 243 (2001). Type: Bolivia, La Paz, Larecaja, between Sorata and Tipuani, 1400 m a.s.l., Dec. 1922, Buchtien 7214 (holo-: B, probably destroyed; lecto-, designated by Luer (2004): US!)

Distribution.-Peru (fig. 10), Venezuela, Colombia, Ecuador and Bolivia. WCSP (2016) gives also Brazil, however according to Barros \& al. (2016) this species does not exist in Brazil.

Comments.-After the clarification of several taxa originally described on the Peruvian expedition of Ruiz \& Pavón (Pupulin 2012), it states clear that Hulboldtia polystachya Ruiz \& Pav. - A. polystachya (Ruiz \& Pav.) Pupulin - represents the first reference to A. cassapensis and should be considered the first available name for the species. In the present work, we follow that conclusion and added a new synonym, A. phyllostachys, treated as different species by Luer on his monograph of the genus and cited by WCSP (2016) as a synonym of $A$. polystachya without any proper reference. Both, A. casapensis and A. phyllostachys share several vegetative features, including shortly repent habit, compressed above the middle ramicauls and elliptical decurrent leaves. Moreover, the only significant difference in what concern floral morphology between these two species are the longer floral segments of A. phyllostachys -i.e., sepals $13 \times 3 \mathrm{~mm}$ vs. $10 \times 3 \mathrm{~mm}$, petals $5 \times 2 \mathrm{~mm}$ vs. $3 \times 1.5 \mathrm{~mm}$, lip 6 $\times 3 \mathrm{~mm}$ vs. $4 \times 2 \mathrm{~mm}$.

According to WCSP (2016), A. harpophylla (Rchb. f.) Luer is listed as a synonym of $A$. polystachya, however this is not fully accurate. Acianthera harpophylla is easily distinguished from A. polystachya by its dorsal sepal, longer than the synsepal - vs. equal length - and its conspicuous intramural calli of the lip with a longitudinal callus from the base to near the apex — vs. low parallel subverrucose calli without a longitudinal callus.

Studied specimens.-PERU. Huánuco: Cassapi (Casapi), Mathews 1830 (K). Amazonas: Bongara, Sipabamba, Quebrada Fortuna, Young and Eisenberg 317 (MO); Chiriaco, van der Werff et al. 24616 (MO). Cajamarca: Chota, Llama-Huambos, forest above Huarimarca, Sagastegui and Leiva 15990 (HAO, MO). Junín: Yaupe, Woytkowski 6402 (MO). Cusco: Calca, quebrada de Cochocc, Vargas 16811 (CUZ!); Calca, proximidades de Hierbabuenayoc, Tupayachi H. 1560 (CUZ!). Pasco: Oxapampa, Dist. Huancabamba, Parque Nacional Yanachaga-Chemillen, Sector Quebrada Yanachaga, Vásquez et al. 30504 (USM!); ibid., Vásquez et al. 29525 (HOXA!); ibid., Vásquez et al. 29236 (HOXA!); Dtto Chontabamba, Cerro Mirador, 2520 m a.s.l., Valenzuela and Mateo 13128 (HOXA!). Piura: Huancabamba, east of Huancabamba, Weberbauer 6313 (AMES). Amazonas: Bongara, Sipabamba, Quebrada Fortuna, Young and Eisenberg 317 (MO). Cajamarca: Chota, Llama-Huambos, Sagastegui and Leiva 15990 (F, HAO, MO). Junín: Yaupe, Woytkowski 6402 (MO). Without locality: Bennet 573 (UC).
24. A. pubescens (Lindl.) Pridgeon \& M.W.Chase, Lindleyana 16 (4): 245-246 (2001); Pleurothallis pubescens Lindl., Compan. Bot. Mag. 2: 355 (1836); Humboltia pubescens (Lindl.) Kuntze, Revis. Gen. Pl. 2: 668 (1891). Type: Mexico, s.loc., Barker s.n. (holo-: K, not located, drawing at AMES 74658!).

Pleurothallis vittata Lindl., Edwards's Bot. Reg. 24 (Misc.): 73 (1838); Humboltia vittata (Lindl.) Kuntze, Revis. Gen. Pl. 2: 668 (1891). Type: Mexico, s.loc., Loddiges s.n. (holo-: K).

Pleurothallis smithiana Lindl., Edwards's Bot. Reg. 29 (Misc.): 57 (1843); Humboltia smithiana (Lindl.) Kuntze, Revis. Gen. Pl. 2: 668 (1891) Type: Brazil, Rio de Janeiro, Dec. 1841, Smith s.n. (holo-: K!).

Pleurothallis polystachya A.Rich. \& Galeotti, Ann. Sci. Nat., Bot., sér. 3, 3: 16 (1845); Humboltia polystachya (A.Rich. \& Galeotti) Kuntze, Revis. Gen. Pl. 2: 668 (1891), nom. illegit., non Ruiz \& Pav., Syst. Veg. Fl. Peruv. Chil.: 234 (1798). Type: Mexico, Veracruz, 1840, Galeotti 5135 (holo-: W; iso-: G!)

Pleurothallis bufonis Klotzsch, Allg. Gartenzeitung 22: 225 (1854); Humboltia bufonis (Klotzsch) Kuntze, Revis. Gen. Pl. 2: 667 (1891). Type: Venezuela, Trujillo, Wagener s.n. (holo-: W, not located).

Pleurothallis truxillensis Rchb. f., Bonplandia (Hannover) 2: 25 (1854); Humboltia truxillensis (Rchb. f.) Kuntze, Revis. Gen. Pl. 2: 668 (1891). Type: Venezuela, Trujillo, $6000 \mathrm{ft}$ a.s.l., Dec. 1850, Wagener 31 (holo-: W, not located).

Pleurothallis janeirensis Barb.Rodr., Gen. Spec. Orchid. 2: 29 (1881). Type: Brazil, Rio de Janeiro, Rodeio, Barbosa s.n. (holo-: RB, lost; lecto-, here designated: Sprunger 1996: 227 tab. 169B).

Pleurothallis rio-grandensis Barb.Rodr., Gen. Spec. Orchid. 2: 28 (1881). Type: Brazil, Rio Grande do Sul, Barbosa s.n. (holo-: RB, lost; lecto-, here designated: Sprunger 1996: 227 tab. 169A).

Pleurothallis janeirensis var. viridicata Barb.Rodr., Gen. Spec. Orchid. 2: 30 (1881); Pleurothallis smithiana var. viridicata Cogn. in Mart., Fl. Bras. 3 (4): 533 (1896). Type: Brazil, Rio de Janeiro, Oriente, Barbosa s.n. (holo-: RB, lost).

Pleurothallis coriacea Bello, Anales Soc. Esp. Hist. Nat. 12: 116 (1883). Type: Puerto Rico, Bello s.n. (holo-: B, destroyed). Puerto Rico, near Adjuntas in monte Bello, 9 Apr. 1886, Sintenis 4251 (neo-, designated by Luer (2004): AMES!)

Pleurothallis rio-grandensis var. longicaulis Cogn. in Mart., Fl. Bras. 3 (4): 542 (1896). Type: Brazil, Rio de Janeiro, Rodeio, Barbosa s.n. (holo-: RB, lost).

Pleurothallis mandibularis Kraenzl., Vidensk. Meddel. Naturhist. Foren. Kjøbenhavn 71: 169 (1920). Type: Mexico, Veracruz, Liebmann s.n. (holo-: C, drawing at AMES 74462!).

Pleurothallis bourgeaui Kraenzl., Ark. Bot. 16 (8): 15 (1921). Type: Mexico, Veracruz, Orizaba, 17 May 1869, Bourgeau 2469 (holo-: P!; iso-: MPU!, G!).

Pleurothallis porphyrantha Kraenzl., Ark. Bot. 16 (8): 10 (1921). Type: Brazil, Paraná, Itarare, Dusen 11573 (holo-: HBG!; iso-: AMES!)

Distribution.-Bolivia, Brazil, Caribbean, Colombia, Costa Rica, Ecuador, French Guiana, Guatemala, Honduras, Mexico, Uruguay, Nicaragua, Panama, Peru (fig. 11), Suriname and Venezuela.

Comments.-Acianthera pubescens is a widely distributed species ranging from Mexico to Central America - Honduras, Costa Rica and Panama-, all through the Andes - Peru, Colombia and Bolivia — and into southern Brazil and Argentina. Some differences have been suggested for populations of Central America and Mexico leading to the segregation of species traditionally considered as synonyms and the recognition of very similar species as distinct —e.g., A. breedlovei and A. majoluckae — (Solano 2015; Soto \& al. 2002). We believe those differences fit on the broad concept of A. pubescens as Luer (2004) proposed. Solano (2015) justified its segregation based mainly on vegetative features such as leaves and 
stem lenght, while other taxonomically relevant attributes such as petals and lip shape remain constant. Further studies are required to attend if variation among geographically separate populations of $A$. pubescens could justify its partition into an intra-specific level as subspecies as we suspect.

Peruvian specimens of $A$. pubescens have narrower leaves -10-12 $\times 1-3 \mathrm{~cm}$ - , long inflorescences $-5-7 \mathrm{~cm}-$ and flowers dimension about the average - sepals 5-7 $\times 2-5 \mathrm{~mm}$; petals $2-3 \times 1-2 \mathrm{~mm}$; long lip $3 \mathrm{~mm}$ - In addition, these specimens grow as epiphytes in cloud forests between $800-2500 \mathrm{~m}$ a.s.l., something atypical for the species, since most of the material proceed from drier areas.

Studied specimens.-PERU. San Martín: San Roque, Williams 7259 (AMES, F); Lamas, Alfonso de Alvarado, road to Moyobamba, Schunke 6031 (AMES, NY). Pasco: Oxapampa, Bennett 1510 (AMES, UC). Huancavelica: Tajacaya, Huachocolpa, Leon et al. 2981 (MOL!).

25. A. rodolfo-vasquezii Damian, Brittonia (2018). Type: Peru, Oxapampa, Estación Biológica Jardín Botánico de Missouri, Orquidiario, área de cultivo en JBM, $2000 \mathrm{~m}$ a.s.1., Vásquez et al. 36591 (holo-: USM!; iso-: HOXA!). Fig. 2d.

\section{Distribution.-Endemic to Peru (fig. 11).}

Studied specimens.-PERU. Amazonas: Utcubamba, Dtto. Bagua Grande, Caserio Berlin, Bosque Berlin, 5 53' 53.47" S, 78 25' 31.10" W, 1900 m a.s.l., Damián and Rimarachin 01165 (UFV!). Huánuco: Chinchao, San Pedro de Carpish, cerca al rio Lan manio, que divide al distrito de Churubamba de Carpish, a la localidad de Carpish, Salinas and Chocce 510 (USM!). Pasco: Dtto Huancabamba, Parque Nacional Yanachaga-Chemillen, bosque remante parcialmente intervenido, $2265 \mathrm{~m}$ a.s.1., Vásquez et al. 30502 (USM!); Oxapampa, Dtto Chorobamba, $1700 \mathrm{~m}$ a.s.1., Smith and Brak 7631 (USM!).

26. A. rubroviridis (Lindl.) Pridgeon \& M.W.Chase, Lindleyana 16 (4): 246 (2001); Pleurothallis rubroviridis Lindl., Ann. Mag. Nat. Hist. ser. 3, 1: 327 (1858); Humboltia rubroviridis (Lindl.) Kuntze, Revis. Gen. P1. 2: 668 (1891). Type: Cuba, Orientali, 1856-1857, Wright s.n. (holo-: K!). Fig. 2e.

Pleurothallis cubensis Lindl., Ann. Mag. Nat. Hist. ser. 3, 1: 328 (1858); Humboltia cubensis (Lindl.) Kuntze, Revis. Gen. Pl. 2: 667 (1891); Acianthera cubensis (Lindl.) Pridgeon \& M.W.Chase, Lindleyana 16:

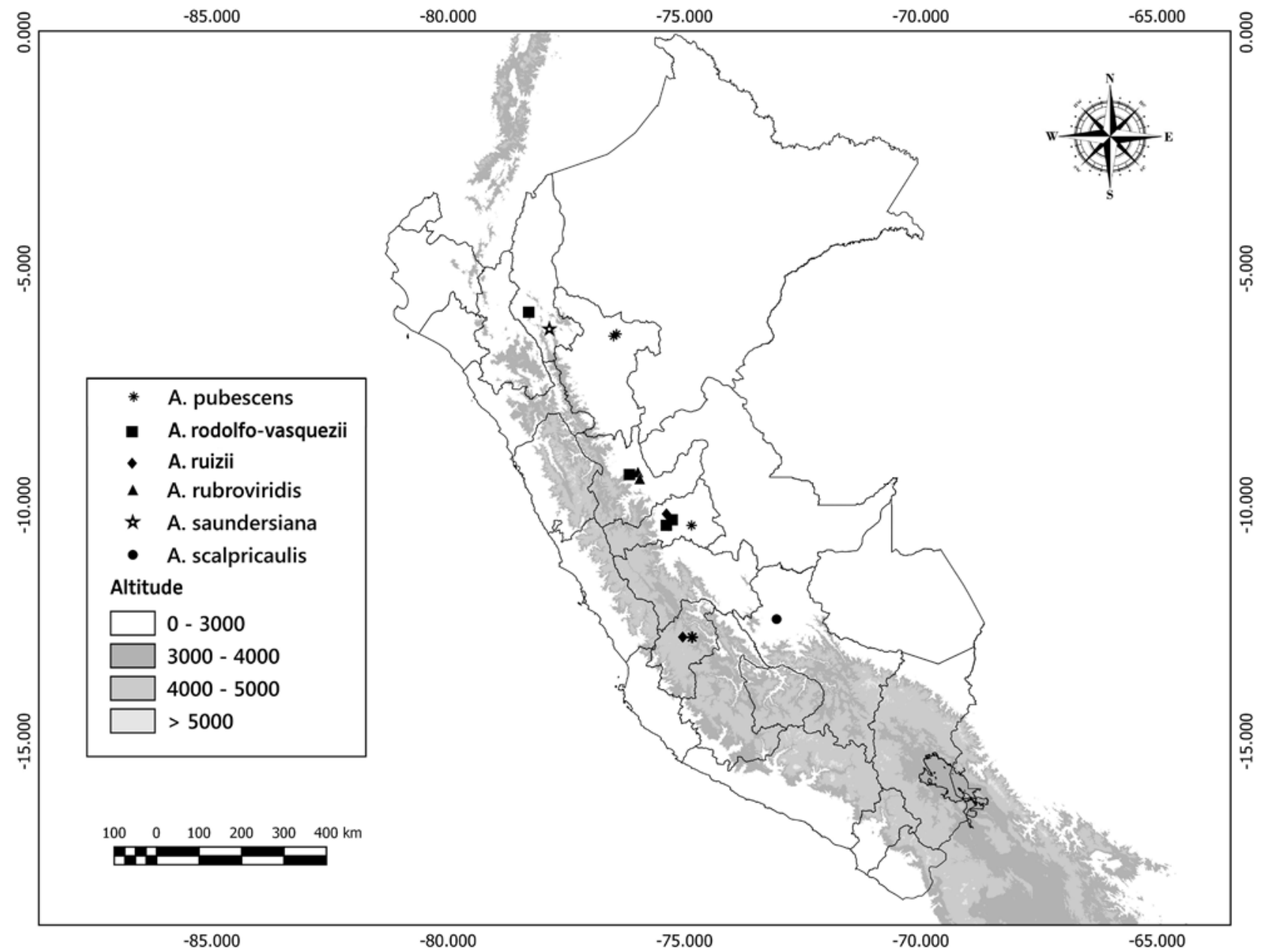

Fig. 11. Map showing the known Peruvian localities for Acianthera pubescens (Lindl.) Pridgeon \& M.W.Chase, A. rodolfo-vasquezii Damian, A. ruizii Damian, A. rubroviridis (Lindl.) Pridgeon \& M.W.Chase, A. saundersiana (Rchb. f.) Pridgeon \& M.W.Chase and A. scalpricaulis (Luer) Pridgeon \& M.W.Chase. 
243 (2001). Type: Cuba, Monte Verde, 1 Jun. 1859, Wright 653 (holo-: $\mathrm{K}$ !; iso-: AMES!, LE!, $\mathrm{P}$ !).

Pleurothallis verruculosa Kraenzl., Repert. Spec. Nov. Regni Veg. 1: 177 (1905). Type: Peru, Cajamarca, Chota, west of Huambos, 3100 m a.s.1., Weberbauer 4180 (holo-: B, lost; lecto-, designated here: AMES!, 38671-barcode).

Distribution.-Caribbean, Ecuador, Peru (fig. 11) and Venezuela.

Studied specimens.-PERU. Huánuco: Tingo Maria, E. Asplund 12165 (AMES, S); South of Tingo María, Río Huallaga, Luer et al. 5341 (SEL).

27. A. ruizii Damian, Brittonia (2018). Type: Peru, Oxapampa, Parque Nacional Yanachaga Chemillen, 2024 m a.s.l., Perea et al. 4127 (holo-: USM!, iso-: HOXA!).

Distribution.-Endemic to Peru (fig. 11).

Studied specimens.-PERU. Huancavelica: Tajacaya, Dtto. Huachocolpa, Capcasorco, Leon 2962 (MOL!).

28. A. saundersiana (Rchb. f.) Pridgeon \& M.W.Chase. Lindleyana 16 (4): 246 (2001); Pleurothallis saundersiana
Rchb. f., Gard. Chron. 1866: 74 (1866); Specklinia saundersiana (Rchb. f.) F.Barros, Hoehnea 10: 110 (1984). Type: Brazil, s.loc. Saunders s.n [cult. at Hillfield House, near Reigate] (lecto-, designated by Toscano \& Luer (2015): W!).

Pleurothallis felis-lingua Barb.Rodr., Gen. Spec. Orchid. 2: 18 (1881). Type: Brazil, Rio de Janeiro, Rodeio, Barbosa s.n. (holo-: RB, lost; lecto-, designated by Chiron \& Bolsanello (2010): tab. 158A).

Pleurothallis josephensis Barb.Rodr., Vellosia ed. 2, 1: 117 (1891). Type: Brazil, Minas Gerais, Serra de São José d'El Rey, Barbosa s.n. (holo-: RB, lost; lecto-, designated by Chiron \& Bolsanello (2010): tab. 160E).

Pleurothallis repens Rolfe, Bull. Misc. Inform. Kew 1912: 131 (1912), nom. illegit., non Ames, Orchidaceae 2: 271-272 (1908); Pleurothallis ascendens Garay, Arch. Jard. Bot. Rio de Janeiro 12: 171 (1953). Type: Brazil, Bahia, s.loc., Wigan s.n. (holo-: K).

Pleurothallis juergensii Schltr., Repert. Spec. Nov. Regni Veg. Beih. 35: 54 (1925). Type: Brazil, Rio Grande do Sul, Rio Pardo, fazenda Boa Esperanza, $70 \mathrm{~m}$ a.s.1., Mar. 1921, Jürgens 20 (holo-: B, destroyed).

Pleurothallis auriculigera Hoehne \& Schltr., Arch. Bot. São Paulo 1: 207 (1926), nom. illegit., non (Rchb. f.) Rchb. f., Gard. Chron. 1871: 1579 (1871). Type: Brazil, São Paulo, Pirajussára, 14 Jun. 1921, Gehrt 5713 (holo-: HB!; iso-: AMES!, SP!).

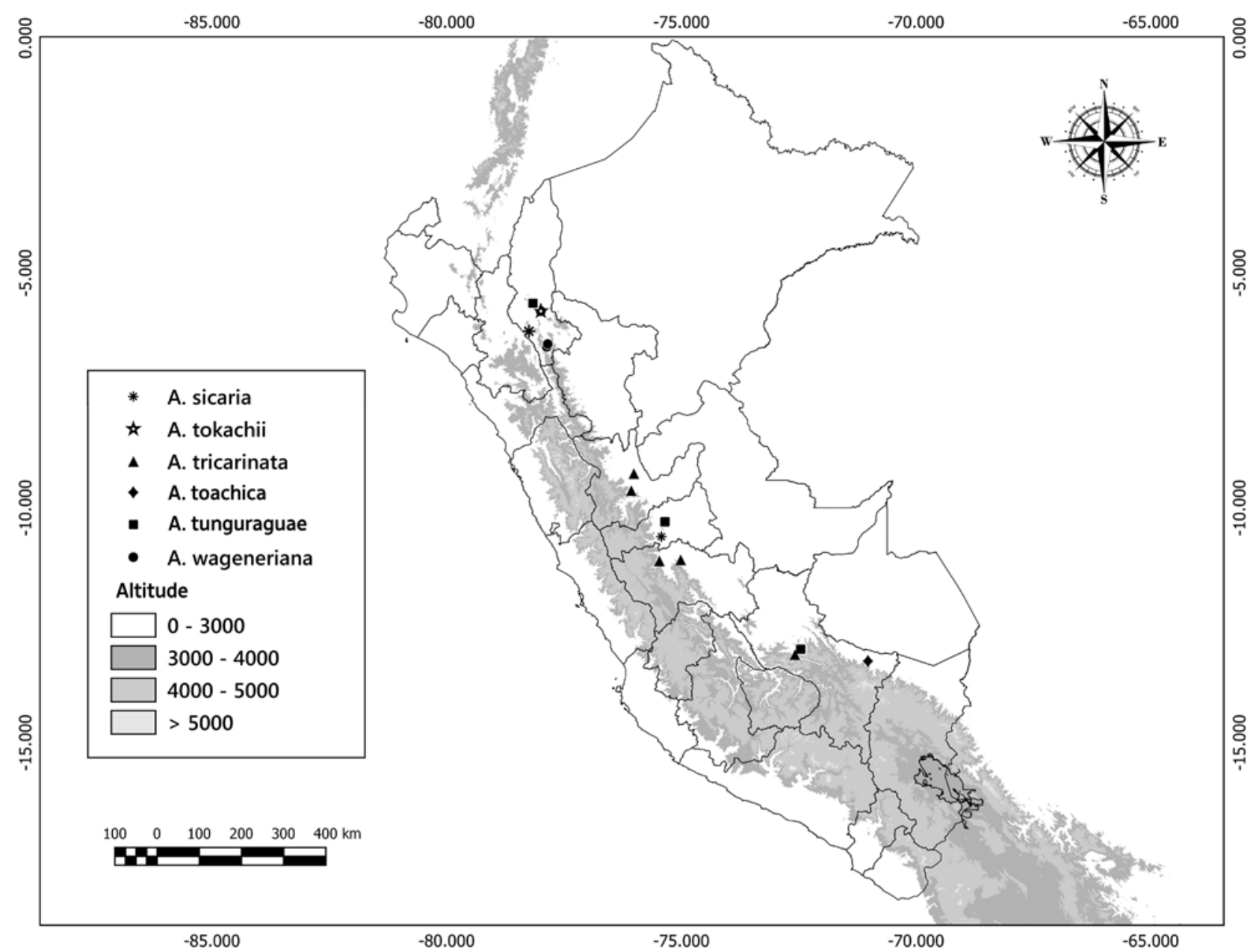

Fig. 12. Map showing the known Peruvian localities for Acianthera sicaria (Lindl.) Pridgeon \& M.W.Chase, A. tokachii (Luer) Luer, A. tricarinata (Poepp. \& Endl.) Pridgeon \& M.W.Chase, A. toachica (Luer \& Dodson) Luer, A. tunguraguae (F.Lehm. \& Kraenzl.) A.Doucette and A. wageneriana (Klotzsch) Pridgeon \& M.W.Chase. 
Pleurothallis butantanensis Hoehne \& Schltr., Arch. Bot. São Paulo 1: 209 (1926). Type: Brazil, São Paulo, Butantan, 20 Jun. 1921, Hoehne s.n. (holo-: B, destroyed; lecto-, designated by Luer (2004): SP 5718!; isolecto-: HB!).

Pleurothallis insularis Hoehne \& Schltr., Arch. Bot. São Paulo 1: 217 (1926); A. insularis (Hoehne \& Schltr.) Luer, Monogr. Syst. Bot. Missouri Bot. Gard. 112: 118 (2007). Type: Brazil, São Paulo, Ilha da Queimada, Gehrt 5452 (holo-: B, destroyed; lecto-, designated by Barros (2004): $\mathrm{SP} !)$

Distribution.-Bolivia, Colombia, Peru (fig. 11) and Brazil.

Comments.-For a detailed revision of this species refer to Chiron \& Bolsanello (2010) and Toscano \& Luer (2015).

Studied specimens.-PERU. Amazonas: near Chachapoyas, Tokach 17 (MO).

29. A. scalpricaulis (Luer) Pridgeon \& M.W.Chase, Lindleyana 16 (4): 246 (2001); Pleurothallis scalpricaulis Luer., Selbyana 1 (4): 424, fig. 103 (1976). Type: Ecuador, Morona-Santiago, about $30 \mathrm{~km}$ south of Gualaquiza, $500 \mathrm{~m}$ a.s.1., 6 Aug. 1975, Luer et al. 619 (holo-: SEL). Fig. 2f.

Distribution.-Bolivia, Ecuador and Peru (fig. 11).

Comments.-Also reported for Huánuco by Blas (2015: 45) as Acianthera sp.

Studied specimens.-PERU. Cusco: La Convención, about $5 \mathrm{~km}$ from Ivochote valley, Damián and Huaycho 1086 (UFV!).

30. A. sicaria (Lindl.) Pridgeon \& M.W.Chase, Lindleyana 16 (4): 246 (2001); Pleurothallis sicaria Lindl., Edwards's Bot. Reg. 27 (Misc.): 91 (1841); Humboltia sicaria (Lindl.) Kuntze, Revis. Gen. Pl. 2: 668 (1891). Type: Trinidad and Tobago, s.loc., Loddiges s.n. (holo-: K!). Fig. $2 \mathrm{~g}$.

Pleurothallis tripteris Rchb. f., Linnaea 22: 829 (1850). Type: Venezuela, Districto Federal, Caracas, 4000 ft a.s.1., Jan. 1846, Schlim 127 (holo$\mathrm{W}$; iso-: $\mathrm{P}$ !).

Pleurothallis trigonopoda Klotzsch, Allg. Gartenzeitung 21: 361 (1853) Type: Venezuela, Aragua, s.loc., Wagener s.n. (holo-: unknown).

Pleurothallis alpina Ames, Schedul. Orchid. 5: 14 (1923); A. alpina (Ames) Pridgeon \& M.W.Chase, Lindleyana 16: 242 (2001). Type: Panama, Chiriqui, «Caramilla», $1500 \mathrm{~m}$ a.s.1., Mar. 1923, Powell 282 (holo-: AMES!; iso-: MO!)

Distribution.-Bolivia, Caribbean, Colombia, Costa Rica, Ecuador, French Guiana, Guyana, Panama, Peru (fig. 12), Suriname, Trinidad and Venezuela.

Studied specimens.-PERU. Amazonas: Luya, Ocalli, anexo Ouise, Diaz et al. 4400 (USM!, MO). Pasco: Oxapampa, Dist. Huancabamba, Sector Tunqui, camino a Barro Blanco, Cardenas et al. 0968 (USM!, HOXA!).

31. A. toachica (Luer \& Dodson) Luer, Monogr. Syst. Bot. Missouri Bot. Gard. 95: 254 (2004); Pleurothallis toachica Luer \& Dodson, Lindleyana 11: 188 (1996). Type: Ecuador, Pichincha, between Chiriboga and Santo Domingo, Apr. 1986, Dodson and D'Alessandro $16374^{a}$ (holo-: MO!). Fig. 2 h.

Distribution.-Ecuador and Peru (fig. 12).

Studied specimens._PERU. Cusco: Quispicanchis, Camanti, Nov. 2009 Ballon et al. 62 (BRIT!).
32. A. tokachii (Luer) Luer, Monogr. Syst. Bot. Missouri Bot. Gard. 95: 254 (2004); Pleurothallis tokachii Luer, Lindleyana 12 (1): 55, fig. 14 (1997). Type: Peru, Amazonas, near Pomacochas, Feb. 1993, Tokach p-16 (holo-: MO!). Fig. 2 i.

Distribution.-Endemic to Peru, known only from the type locality (fig. 12).

33. A. tricarinata (Poepp. \& Endl.) Pridgeon \& M.W.Chase, Lindleyana 16 (4): 246 (2001); Pleurothallis tricarinata Poepp. \& Endl., Nov. Gen. Sp. Pl. 1: 49-50, pl. 87 (1836); Humboltia tricarinata (Poepp. \& Endl.) Kuntze, Revis. Gen. P1. 2: 668 (1891). Type: Peru, Huánuco, Cuchero, Feb. 1830, Poeppig 1733 t.87 (holo-: W!). Fig. 2j.

Pleurothallis acutangula H.Wendl. \& Kraenzl. in Rchb. f., Xenia Orchid. 3: tab. 298 (1900). Type: Brazil, Minas Gerais, s.loc., Wendland s.n. [cult.] (holo-: B, destroyed).

Pleurothallis trialata Rolfe, Bull. New York Bot. Gard. 4: 449 (1907), nom. illegit., non Cogn. in Mart., Fl. Bras. 3 (4): 500-501, tab. 100, fig. 2 (1896); Pleurothallis triptera Schltr., Repert. Spec. Nov. Regni Veg. Beih. 10: 68 (1922). Type: Bolivia, s.loc., Bang 1816 a (holo-: NY).

Distribution.-Bolivia, Ecuador and Peru (fig. 12).

Studied specimens._PERU. Huánuco: above Tingo Maria, Jara B-4214 (MO). Cusco: Urubamba, Vargas 3403 (AMES, CUZ). Junín: Shunke Hacienda, above San Ramón, Schunke A. 56 (F); ibid., Schunke 1091, $1185(\mathrm{~F} !)$.

34. A. tunguraguae (F.Lehm. \& Kraenzl.) A.Doucette, Phytotaxa 275 (3): 263-276 (2016); Otopetalum tunguraguae F.Lehm. \& Kraenzl., Bot. Jahrb. Syst. 26: 457 (1899); Kraenzlinella tunguraguae (F.Lehm. \& Kraenzl.) Kuntze ex Engl. \& Prantl in Engl. \& Prantl (eds.), Nat. Pflanzenfam. 3: 86 (1906); Pleurothallis otopetalum Schltr., Repert. Spec. Nov. Regni Veg. 10: 292 (1912); Kraenzlinella otopetalum (Schltr.) Luer, Monogr. Syst. Bot. Missouri Bot. Gard. 95: 258 (2004), nom. superfl. Type: Ecuador, Tungurahua, Volcan Tungurahua near Baños, Lehmann 8088 (holo-: K!; iso-: AMES!). Fig. 2k.

Distribution.-Ecuador, Colombia and Peru (fig. 11).

Studied specimens.-PERU. Amazonas: Bongara, near Shilla, K. Young and Eisenberg 420 (MO); Bagua, Bosque Berlin, Damián and Rimarachin 01124 (UFV!). Pasco: south of Oxapampa, C. Luer and J. Luer 3841 (SEL). Cusco: Urubamba, Aguas Calientes, Nuñez 7543 (MO).

35. A. wageneriana (Klotzsch) Pridgeon \& M.W.Chase, Lindleyana 16 (4): 246 (2001); Pleurothallis wageneriana Klotzsch, Allg. Gartenzeitung 20: 274 (1852). Type: Venezuela, Caracas, Wagener 344 (holo-: W). Fig. 21.

Pleurothallis convexifolia Barb.Rodr., Gen. Spec. Orchid. 1: 17 (1877). Type: Brazil, Minas Gerais, Caldas, Barbosa s.n. (holo-: RB, lost; lecto-, here designated: Sprunger 1996: 228 tab. 170).

Pleurothallis xylobiochila Kraenzl., Ark. Bot. 16 (8): 9 (1921). Type: Brazil, Paraná, near Jaguariahyva, 12 Mar. 1911, Dusen 11583 (holo-: S!; iso-: AMES!).

Pleurothallis dasychila Luer, Selbyana 3: 286 (1977); A. dasychila (Luer) Carnevali \& G.A.Romero in O.Hokche \& al., Nuevo Cat. Fl. Vasc. 
Venezuela: 753 (2008). Type: Ecuador, Loja, road north of Loja, $2000 \mathrm{~m}$ a.s.1., 5 Mar. 1977, Luer et al. 1545 (holo-: SEL!).

Distribution.-Colombia and Peru (fig. 12).

Studied specimens.-PERU. Amazonas: Prov. of Bongara, vicinity of Campamiento Ingenio, Hutchison and Wright 3832 (USM!, AMES, UC) between Pomacocha and Campamento Ingenio, Hutchison and Wright 4020 (USM!); between Río Utcubamba and lake Pomacochas on the Rioja road, Hutchison and Wright 6840 (MO); Bagua, Bosque Berlín, 1900 m a.s.1., Rimarachin 508 (HUT!).

\section{Excluded species}

A. sicariopsis (Luer) Pridgeon \& M.W.Chase, Lindleyana 16 (4): 246 (2001); Pleurothallis sicariopsis Luer, Phytologia 49 (3): 217 (1981). Type: Ecuador, Cotopaxi, west of El Corazón, 1200 m a.s.1., 18 Feb. 1979, Luer et al. 4025 (holo-: SEL!).

Distribution.-Ecuador and Bolivia.

Comments.-This taxon is cited for Peruvian flora by Bracko \& Zarucchi (1993) without any voucher information. According to Luer (2004) this species is endemic to Ecuador. Although WCSP (2016) gave a distribution for this species from Ecuador to Bolivia through Peru, there is no evidence for the presence of the species in Peru.

\section{ACKNOWLEDGMENTS}

The authors would like to thank the staff and curators from USM, MOL and HOXA for allowing access to their collections; Ruben Sierra for assisting with literature; Erica Morón Abad, Luis Filipe Varela, Luis Egoavil, Jose Edquen, Luis Ocupa, Sofia Arevalo, John Janovec, Sergio Olortegui, Luis Torres and K. Yupanqui for sharing their photos of Acianthera; Walter Espindola for elaborating the distribution maps; Leyda Rimarachin and her family for field work support during our visit to Berlin-Amazonas; and the reviewers for their valuable recommendations on the manuscript, specially to Rodolfo Solano who provided critical additional comments and corrections.

\section{REFERENCES}

Barringer K.A. 1986. Typification of Schlechter's Costa Rican Orchidaceae. I, Types collected by A. Brenes. Chicago Natural History Museum, Chicago. https://doi.org/10.5962/bhl.title.2664

Barros F. 2002. Notas nomenclaturais em Pleurothallidinae (Orchidaceae), principalmente brasileiras. Bradea 8: 293-297.

Barros F. \& Guimarães L.R.S. 2010. New combinations and a new name in Brazilian Orchidaceae. Neodiversity 5: 26-33. https://doi.org/10.13102/neod.51.7

Barros V.F., Rodrigues V.T., Barberena F.F.V.A., Fraga C.N., Pessoa E.M., Forster W., Menini Neto L., Furtado S.G., Nardy C. \& Azevedo C.O., Guimarães L.R.S. 2016. Orchidaceae in Lista de Espécies da Flora do Brasil. Website: http://floradobrasil.jbrj.gov.br/jabot/floradobrasil/FB10986 [accessed: 18 Feb. 2016].

Bennett D.E.J. \& Christenson E.A. 1993-2001. Icones Orchidacearum Peruvianum [plates 1-600]. David Bennett, Lima.

Blas J. 2015. Diversidad, Rescate y Conservación de Orquídeas, en el proyecto Central Hidroeléctrica Chaglla. Centro de Producción Fondo Editorial Universidad Nacional Mayor de San Marcos, Lima.

Brako L. \& Zarucchi J. 1993. Catalogue of the Flowering Plants and Gymnosperms of Peru. Monographs in Systematic Botany of the Missouri Botanical Garden 45: 414-425.
Chase M.W., Cameron K.M., Freudenstein J.V., Pridgeon A.M., Salazar G., van den Berg C. \& Schuiteman A. 2015. An updated classification of Orchidaceae. Botanical Journal of the Linnean Society 177: 151-174. https://doi.org/10.1111/boj.12234

Chiron G.R., Guiard J. \& van den Berg C. 2012. Phylogenetic relationships in Brazilian Pleurothallis sensu lato (Pleurothallidinae, Orchidaceae): evidence from nuclear ITS rDNA sequences. Phytotaxa 46: 34-58. https://doi.org/10.11646/phytotaxa.46.1.5

Chiron G.R. \& van den Berg C. 2012. Révision taxonomique du genre Acianthera (Orchidaceae, Pleurothallidinae). Richardiana 12: 59-77.

Chiron G.R. \& Bolsanello R.X. 2010. Quatre taxons nouveaus pour 1'Éspírito Santo (Brésil) dans le morphogroupe Acianthera saundersiana (Orchidaceae). Richardiana 10 (4): 198-220.

Damián A. 2018. Acianthera rodolfo-vasquezii and A. ruizii, spp. nov. (Orchidaceae: Pleurothallidinae) from the montane cloud forest of the central Andes, Peru. Brittonia 2018: 1-6. https://doi.org/10.1007/s12228-017-9508-y

Karremans A., Bogarin D., Diaz-Morales M., Fernandez M., Oses L. \& Pupulin F. 2016. Phylogenetic Reassessment of Acianthera (Orchidaceae: Pleurothallidinae). Harvard Papers in Botany 21 (2): 171-187. https://doi.org/10.3100/hpib.v21iss2.2016.n4

León B., Roque J. \& Ulloa Ulloa C. 2006. Libro Rojo de las Plantas endémicas del Perú. Revista Peruana de Biología, Número Especial 13 (2): 1-971.

Lindley J. 1859. Pleurothallis. Folia Orchidacea: an enumeration of the known species of Orchids. J. Mathews, London.

Luer C.A. 1986. Icones Pleurothallidinarum 3: Systematics of Pleurothallis. Monographs in Systematic Botany of the Missouri Botanical Garden 20: 1-120.

Luer C.A. 2002. A Systematic method of Classification of the Pleurothallidinae Versus a Strictly Phylogenetic Method. Selbyana 23 (1): 57-110.

Luer C.A. 2004. Icones Pleurothallidinarum 26: Pleurothallis subgenus Acianthera and three allied subgenera. A second century of new species of Stelis of Ecuador, Epibator, Ophidion, Zootrophion. Monographs in Systematic Botany of the Missouri Botanical Garden 95: 1-265.

Luer C.A. 2006. Icones Pleurothallidinarum 28: A reconsideration of Masdevallia, Systematics of Specklinia and vegetatively similar taxa (Orchidaceae). Monographs in Systematic Botany of the Missouri Botanical Garden 105: 1-274.

Luer C.A. 2009. Icones Icones Pleurothallidinarum 30: Lepanthes of Jamaica. Systematis of Stelis of Ecuador, Part Four. Monographs in Systematic Botany of the Missouri Botanical Garden 115: 1-264.

Luer C.A. 2011. Miscellaneous New Species in the Pleurothallidinae (Orchidaceae) Excluding Species from Brazil. Harvard Papers in Botany 16 (2):311-360. https://doi.org/10.3100/0.25.016.0206

Luer C.A. \& Thoerle L. 2012. Miscellaneous new species in the Pleurothallidinae (Orchidaceae). Harvard Papers in Botany 17: 333368. https://doi.org/10.3100/025.017.0214

McNeill J., Barrie F.R., Buck W.R., Demoulin V., Greuter W., Hawksworth D.L., Herendeen P.S., Knapp S., Mar-hold K., Prado J., Prud'homme van Reine W.F., Smith G.F., Wiersema J.H. \& Turland N.J. 2012. International Code of Nomenclature for Algae, Fungi, and Plants (Melbourne Code). Koeltz, Königstein.

Pridgeon A.M., Solano R. \& Chase M.W. 2001. Phylogenetic relationships in Pleurothallidinae (Orchidaceae): combined evidence from nuclear and plastid DNA sequences. American Journal of Botany 88: 2286-2308. $\underline{\text { https://doi.org/10.2307/3558390 }}$ 
Pridgeon A.M. \& Chase M.W. 2001. A phylogenetic reclassification of the Pleurothallidinae (Orchidaceae). Lindleyana 16: 235-271.

Pridgeon A.M. 2005. Subtribe Pleurothallidinae. In Prigeon A.M., Cribb P.J., Chase M.W. \& Rasmussenp F.M. (eds.), Genera Orchidacearum. Epidendroideae (Part One) 4: 319-422. Oxford University, Oxford.

Pupulin F. 2012. The Orchidaceae of Ruiz \& Pavón's “Flora Peruviana et Chilensis". A taxonomic study. II. Anales del Jardín Botánico de Madrid 69: 143-186. https://doi.org/10.3989/ajbm.2336

Schweinfurth C. 1958-1959. Orchids of Peru. Fieldiana. Botany 30: $1-531$.

Solano R. 2015. A taxonomic synopsis of the Mexican species of Acianthera (Orchidaceae: Pleurothallidinae) including a new species. Phytotaxa 218 (1): 39-60. https://doi.org/10.11646/phytotaxa.218.1.3

Soto M., Solano R. \& Salazar G. 2002. Acianthera breedlovei. In Hagsater E. \& Gerardo A. (eds.), Icones Orchidacearum. Orchids of Mexico 5-6: tab. 502 .

Sprunger S. 1996. João Barbosa Rodrigues - Iconographies des orchidées $d u$ Brésil, v1. The Illustrations. Friedrich Reinhart Verlag, Basle.

Toscano de Brito A. \& Luer C. 2015. New species and nomenclatural notes in Acianthera from Brazil. Lankesteriana 15 (1): 77-92. https://doi.org/10.15517/lank.v15i1.18526

Thiers B. 2017. Index herbariorum: a global directory of public herbaria and associated staff. New York Botanical Garden's virtual herbarium. Website: http://sweetgum.nybg.org/science/ih/ [accessed: Feb. 2017].

Ulloa Ulloa C., Zarucchi J.L. \& León B. 2004. Diez años de adiciones a la Flora del Perú: 1993-2003. Arnaldoa (Edic. Esp. Nov. 2004): 1-242.

WCSP. 2016. World Checklist of Selected Plant Families. The Royal Botanic Gardens, Kew. Website: http://apps.kew.org/wcsp/ [accessed: Feb. 2016].

\section{APPENDIX}

Alphabetical list of collectors included: Andre 4632 (7); Asplund 12165 (26); Becerra et al. 1393 (17); Brenes 27 (16); Berlin 1605 (11); Barker s.n. (21); Bateman s.n. (2); E. Bello s.n. (21); Bennet 573 (23), 1510 (23), 3436 (13); Bourgeau 2469 (21); Barbosa s.n (28), s.n. (18); s.n. (21), s.n. (23); s.n. (2); Ballon et al. 62 (31); Bang $1816 a$ (27); Buchtien 7214 (20); S. B-3856 (2); Calatayud et al. 534, 535 (13); Calatayud and R. Vásquez 520 (13); Calatayud and Vargas 6856 ${ }^{a}, 6848$ (13); Cardenas et al. 0968 (30), 0968 (25); Celedonia 258 (8); Chambi et al. 666 (8);
Croat 17792, 19221, 20402 (17), 18537 (14); Damián 8031 (13); Damián and Mitidieri 03090 (19); Damián and Rimarachin 01166 (3), 1165 (25), 1168 (8), 1124 (34); Damián and Huaycho 1086 (29); Diaz and Osores 2601 (22); Diaz 7655 (17); Diaz et al. 1319 (17), 4400 (30); D'Alessandro 163 (4); Dodson and Thien 889 (8); Dusen 11573 (21), 11583 (28); Focke s.n. (18); Ferreyra 14425 (13); Fernandez and Ruiz 3513 (12); Foster et al. 6731 A (7); Foster 4436 (8); Focke s.n. (15); Gehrt 5452 (23), 5713 (23); Gentry and Revilla 20861 (17); Gentry et al. 76893 (8), 61306 (13), 36521 (8); Galeotti 5135 (21); Hajeck 99, 80 (7), 331 (11), 2196 (13); Hoehne s.n. (23); Hübner 173 (15); Hashimoto and Wada s.n. (12); Hutchison and Kenneth Wright 6841 (7), 3832 (35), 6856A, 3840, 6848 (13), 4020 (35), 6840 (35), 3970 (7); Hutchison and Bennett 4566 (22); Hutchison 6959 (22); Henchman s.n. (15); Householder et al. 769 (17); Herzog 1627; Jara s.n. (17); Jürgens 20 (23); Jara B-4214 (27), s.n. (15); Kujikat 94 (8); Kiilip and Smith 26998 (21), 24221 (7); Klug 10013 (15), 10013 (15), 10050 (17), 10126 (21); Karsten s.n. (20); Leon et al. 2992 (17), 2981 (24), 2962 (27); Lopez et al. 4282 (7); Luer et al. 619 (24), 11766 (10), 13927 (7), 5341 (26), 1545 (28); Luer and Butcher 1375 (16); Luer 1573 (16), 249S (1), 5892 (1), 2901 (18); Luer and Luer 3841 (34); Lankester 454 (13); Liebmann s.n. (21); Lehman 8202 (16), 8167 (15); Lance s.n. (15); Loddiges 13 (15), s.n. (21), s.n. (25); Linden 639 (20); Monteagudo et al. 10168 (17), 11522 (17); Z. Moscoso 843 (22); Mandon 1132 (1); Mexia 8215 (20); Macbride 3837 (2), 4252 (7), 40555 (7); Mathews 1830 (23); Miers s.n (4); Moore 10050 (1); Maas et al. 6154 (17); McDaniel and Rimachi 27836 (8), 26585 (17); Moron de Abad 3 (10), 4 (4); Nakata 626 (1); Nuñez 7543 (34); Nuñez et al. 15545 (22); Ocupa s.n. (7); Olortegui 1 (6); Otto 911 (20); Perea et al. 4127 (27); Poeppig 1733 (33), 1604 (6); Powell 282 (25); Ruiz and Pavón s.n. (20); Revilla 3590 (17), 4310 (8); Rimarachin 508 (35); Rimachi 3700, 7457 (8); Sagastegui and Leiva 15990 (23); Salas s.n. (7), 128 (16); Salinas and Chocce 510 (25); Sodiro s.n. (20); Smith 363 (16), 4010 (17), 3639 (18); Smith and Brak 7631 (25); Solomon 3540 (8); Schunke 1091, 1185 (27), 162 (21), 6031 (23), 56 (27), 1063 (17), 6891 (8), 7307 (17), 7781 (17), 127 (25); W.W. Saunders s.n. (23); Sieber 206 (4); Spruce 2459 (18), 2724, 2725 (15); Tupayachi 1560 (23); Tokach p-16 (32), 17 (28), P-64 (13); O. Tovar 3771 (22); Ureta et al. 52 (11); Valenzuela and Heredia 12216 (17); Valenzuela and Mateo 13128 (23); Vargas 16811 (23), 18425 (14), 18426 (12), 3403 (27), 3757 (5), 6499 (22), 8977 (5); Vásquez et al. 19020, 36508, 34764 (17), 5305 (8), 30504, 29525, 29236 (23), 36591 (25), 30502 (25), 36659, 29638 (11); Vásquez and D. Criollo 5773 (8); Vásquez and Jaramillo 16628, 4890 (8), 8702 (13); Weberbauer 6313 (23); Wright 653 (22), s.n. (22); Wigan s.n. (23); Woytkowski 6402 (23); van der Werff et al. 9920 (17), 24616 (23); Wagener 31 (21), 344 (28), 549 (20), 562 (15), s.n. (21), s.n. (25); Wendland s.n. (27); Williams 7259 (23); Weng 48 (17); Young and M. Eisenberg 317 (23), 420 (34). 